\title{
Cool dust heating and temperature mixing in nearby star-forming galaxies $\star, \star \star, \star \star \star$
}

\author{
L. K. Hunt ${ }^{1}$, B. T. Draine ${ }^{2}$, S. Bianchi ${ }^{1}$, K. D. Gordon ${ }^{3,4}$, G. Aniano ${ }^{5,2}$, D. Calzetti ${ }^{6}$, D. A. Dale ${ }^{7}$, G. Helou ${ }^{8}$, \\ J. L. Hinz ${ }^{9}$, R. C. Kennicutt ${ }^{10}$, H. Roussel ${ }^{11}$, C. D. Wilson ${ }^{12}$, A. Bolatto ${ }^{13}$, M. Boquien ${ }^{14}$, K. V. Croxall ${ }^{15}$, \\ M. Galametz ${ }^{16}$, A. Gil de Paz ${ }^{17}$, J. Koda ${ }^{18}$, J. C. Muñoz-Mateos ${ }^{19}$, K. M. Sandstrom ${ }^{20,21}$, M. Sauvage ${ }^{22}$, \\ L. Vigroux ${ }^{23}$, and S. Zibetti ${ }^{1}$ \\ (Affiliations can be found after the references)
}

Received 1 August 2014 / Accepted 16 September 2014

\begin{abstract}
Physical conditions of the interstellar medium in galaxies are closely linked to the ambient radiation field and the heating of dust grains. In order to characterize dust properties in galaxies over a wide range of physical conditions, we present here the radial surface brightness profiles of the entire sample of 61 galaxies from Key Insights into Nearby Galaxies: Far-Infrared Survey with Herschel (KINGFISH). The main goal of our work is the characterization of the grain emissivities, dust temperatures, and interstellar radiation fields (ISRFs) responsible for heating the dust. We first fit the radial profiles with exponential functions in order to compare stellar and cool-dust disk scalelengths, as measured by $3.6 \mu \mathrm{m}$ and $250 \mu \mathrm{m}$ surface brightnesses. Our results show that the stellar and dust scalelengths are comparable, with a mean ratio of 1.04, although several galaxies show dust-to-stellar scalelength ratios of 1.5 or more. We then fit the far-infrared spectral energy distribution (SED) in each annular region with single-temperature modified blackbodies using both variable (MBBV) and fixed (MBBF) emissivity indices $\beta$, as well as with physically motivated dust models. The KINGFISH profiles are well suited to examining trends of dust temperature $T_{\text {dust }}$ and $\beta$ because they span a factor of $\sim 200$ in the ISRF intensity heating the bulk of the dust mass, $U_{\min }$. Results from fitting the profile SEDs suggest that, on average, $T_{\text {dust }}$, dust optical depth $\tau_{\text {dust }}$, and $U_{\min }$ decrease with radius. The emissivity index $\beta$ also decreases with radius in some galaxies, but in others is increasing, or rising in the inner regions and falling in the outer ones. Despite the fixed grain emissivity (average $\beta \sim 2.1$ ) of the physically-motivated models, they are well able to accommodate flat spectral slopes with $\beta \lesssim 1$. An analysis of the wavelength variations of dust emissivities in both the data and the models shows that flatter slopes $(\beta \lesssim 1.5)$ are associated with cooler temperatures, contrary to what would be expected from the usual $T_{\text {dust }}-\beta$ degeneracy. This trend is related to variations in $U_{\min }$ since $\beta$ and $U_{\min }$ are very closely linked over the entire range in $U_{\min }$ sampled by the KINGFISH galaxies: low $U_{\min }$ is associated with flat $\beta \lessgtr 1$. Both these results strongly suggest that the low apparent $\beta$ values (flat slopes) in MBBV fits are caused by temperature mixing along the line of sight, rather than by intrinsic variations in grain properties. Finally, a comparison of dust models and the data show a slight $\sim 10 \%$ excess at $500 \mu \mathrm{m}$ for low metallicity $(12+\log (\mathrm{O} / \mathrm{H}) \lesssim 8)$ and low far-infrared surface brightness $\left(\Sigma_{500}\right)$.
\end{abstract}

Key words. galaxies: ISM - dust, extinction - galaxies: star formation

\section{Introduction}

The interstellar medium (ISM) is both the cradle and the grave of star formation in galaxies. Gas is converted into stars in dense molecular clouds, and is expelled during a star's lifetime through stellar winds and at the end of its evolution through supernovae. Dust grains are created both during stellar evolution and in the ISM itself, and act as a catalyst for molecule formation. Dust also contributes significantly to the ISM energy budget through photoelectric heating. For many years, the complex interplay among dust, gas, and star-formation processes was studied mainly through global properties, but the advent of infrared (IR) and submillimeter (submm) satellites such as Spitzer and Herschel, together with ground-based facilities, has made

\footnotetext{
* Based on Herschel observations. Herschel is an ESA space observatory with science instruments provided by European-led Principal Investigator consortia and with important participation from NASA.

$\star \star$ Appendices are available in electronic form at http: //www . aanda.org

$\star \star \star$ Data are only available at the CDS via anonymous ftp to cdsarc.u-strasbg.fr (130.79.128.5) or via http://cdsarc.u-strasbg.fr/viz-bin/qcat?J/A+A/576/A33
}

possible resolved studies of the dust and gas in large samples of nearby galaxies beyond the Local Group.

Like the stars and the molecular component of the ISM (e.g., Freeman 1970; Bigiel et al. 2008; Leroy et al. 2008), dust is generally distributed in a disk, often with an exponential decline of surface density with radius (Haas et al. 1998; Bianchi 2007; Muñoz-Mateos et al. 2009a; Bianchi \& Xilouris 2011; Verstappen et al. 2013; De Geyter et al. 2013). It is heated both by young stars from recent episodes of star formation, and by the more diffuse interstellar radiation field (ISRF) produced by the quiescent underlying stellar population (e.g., Draine \& $\mathrm{Li}$ 2007). However, the intense ISRF in the bulges of disk galaxies (Engelbracht et al. 2010; Groves et al. 2012; Draine et al. 2014) can mimic, at least to some degree, the ISRF of star formation; consequently, warm dust emission may not necessarily be uniquely associated with recent star formation (e.g., Sauvage \& Thuan 1992; Bendo et al. 2010, 2012; Boquien et al. 2011). Whereas a global approach is usually unable to distinguish between these alternatives, radial profiles of dust properties are a unique diagnostic for understanding how dust is heated. Such processes are important because of the relation of dust heating to star formation, the star-formation history ( $\mathrm{SFH}$ ) of a galaxy, and its structure. 
In this paper, we assess the spatial variations of dust properties in galaxies with radial surface brightness profiles of the sample from Key Insights into Nearby Galaxies: A Far-IR Survey with Herschel (KINGFISH, Kennicutt et al. 2011). Several previous papers have dealt with spatially-resolved dust heating in the KINGFISH sample (e.g., Aniano et al. 2012; Galametz et al. 2012; Kirkpatrick et al. 2014), but they were based on limited numbers of galaxies and pixel-by-pixel analyses. Here, we study the entire KINGFISH sample of 61 galaxies and assess radial gradients through azimuthal averaging. Thus, we can not only sample varying physical conditions within galaxies, comparing dense inner regions to tenuous outer ones, but also probe beyond typical high surface-brightness boundaries because of the increased signal-to-noise ratio $(\mathrm{S} / \mathrm{N})$ made possible by averaging over faint outer isophotes.

The main goal of this paper is the characterization of the grain emissivities, dust temperatures, and the ISRFs responsible for heating the dust, over a wider range of physical conditions than has been possible up to now. We also want to explore evidence of submm emission in excess of what would be expected from standard dust models (e.g., Planck Collaboration XVII 2011; Galliano et al. 2011; Kirkpatrick et al. 2013; Galametz et al. 2014). To achieve these goals, we adopt a multi-pronged approach which comprises several data fits and fits of models themselves: a modified blackbody (MBB) fitting of the farinfrared spectral energy distribution (SED) at each radial data point within a galaxy; fitting the SED at each radius with the physically-motivated models by Draine \& Li (2007, hereafter DL07); and, finally, fitting the best-fit DL07 models with MBBs in the same way as the data were fit. Both sets of MBB fits are two-fold: one with the dust power-law emissivity index $\beta$ left to vary, and another with $\beta$ fixed. Our objective for fitting the DL07 models with MBBs is to connect the physical parameters of the DL07 models with the approximation of a single dust temperature, as well as to assess how well fixed-emissivity models such as DL07 can accommodate apparent values of $\beta$ lower than the intrinsic emissivity index assumed in the model.

The paper is structured as follows: sample selection and ancillary data are described in Sect. 2, together with Herschel image reduction and data preparation. Section 3 explains the extraction of the radial profiles, and the analysis of the disk scalelengths of the dust and stars; Sect. 4 shows SPIRE and PACS colors of the radial profile data. We outline the model fitting of the radial profile SEDs in Sect. 5, and discuss the fitting results in two sections: Sect. 6 (radial trends) and Sect. 7 (emissivity variations, temperature mixing, and assessment of the models). Our conclusions are given in Sect. 8 .

\section{The sample and the data}

The KINGFISH sample includes 61 nearby galaxies within $30 \mathrm{Mpc}$, selected to cover the variety of observed galaxy morphologies, and the range of masses and luminosities within each morphology as well as a variation of dust opacities (Kennicutt et al. 2011). 57 of these galaxies derive from the earlier SIRTF Infrared Nearby Galaxy Survey (SINGS, Kennicutt et al. 2003) which comprised 75 nearby galaxies. Although both samples are heavily biased toward star-forming galaxies, $\sim 16 \%$ of the KINGFISH sample are early types, ellipticals and lenticulars (S0's). Table 1 lists the KINGFISH galaxies, together with some of their observational parameters.

The stellar masses of KINGFISH galaxies span 5 orders of magnitude (from $2 \times 10^{6} M_{\odot}$, DDO 53, to $3 \times 10^{11} M_{\odot}$, NGC 1316) with star-formation rates (SFRs) from $2 \times 10^{-3} M_{\odot} \mathrm{yr}^{-1}$ to $\sim 9 M_{\odot} \mathrm{yr}^{-1}$ (Skibba et al. 2011; Kennicutt et al. 2011). Only NGC 2146 and NGC 1097 might be considered "starbursts" in terms of their specific SFR (SFR divided by stellar mass, sSFR) of $\sim 0.5 \mathrm{Gyr}^{-1}$; most of the remaining galaxies are star-forming galaxies along the "main sequence" of star formation (Salim et al. 2007; Noeske et al. 2007; Karim et al. 2011). Some of the highest SFRs in the KINGFISH sample are found in the lenticulars (e.g., NGC 1482, NGC 1377, and NGC 1266); Such intense star-formation activity is not particularly unusual in S0 galaxies (e.g., Amblard et al. 2014), although these galaxies are not typical early-type systems. NGC 1377 is a nascent starburst with exceptionally warm dust and a deep silicate absorption feature at $9.7 \mu \mathrm{m}$ (Vader et al. 1993; Laureijs et al. 2000; Roussel et al. 2006), and NGC 1266 has shock-excited molecular gas entrained in a molecular outflow (e.g., Alatalo et al. 2011; Pellegrini et al. 2013).

\subsection{Metallicity and metallicity gradients}

Moustakas et al. (2010) presented optical long-slit observations and measured oxygen abundances $(\mathrm{O} / \mathrm{H})$ and their radial gradients for galaxies in the SINGS sample. We adopt these for the KINGFISH galaxies, using the Pilyugin \& Thuan (2005, hereafter PT) calibration (given in Table 1 of Kennicutt et al. 2011). Four KINGFISH galaxies are missing from Moustakas et al. (2010); for these we rely on abundances taken from the literature, which have been reported to the same (PT) metallicity scale. These include IC 342 (Pilyugin et al. 2007), NGC 2146 (Moustakas \& Kennicutt 2006), NGC 3077 (Storchi-Bergmann et al. 1994; Croxall et al. 2009), and NGC 5457 (M 101, Li et al. 2013). Our metallicities for these galaxies are the same as those given by Kennicutt et al. (2011), except for NGC 3077 where we have adopted the determination by Croxall et al. (2009). We incorporate metallicity gradients in the profile analysis when available; Moustakas et al. (2010) gives significant gradients for 17 KINGFISH galaxies. M 101 and IC 342 also have welldetermined metallicity gradients, so that for 19 galaxies we are able to incorporate abundance gradients in our radial analysis. The adopted metallicities are also listed in Table 1 .

\subsection{Herschel PACS and SPIRE data}

The KINGFISH open-time key project acquired far-infrared (FIR) images for 61 galaxies with two instruments on board Herschel (Pilbratt et al. 2010). PACS (Photodetector Array Camera and Spectrometer ${ }^{1}$, Poglitsch et al. 2010) images at $70 \mu \mathrm{m}, 100 \mu \mathrm{m}$ and $160 \mu \mathrm{m}$ were acquired in scan mode at medium speed $\left(20^{\prime \prime} \mathrm{s}^{-1}\right)$ with two orthogonal scans in order to better remove instrumental artefacts and transients. We tailored the PACS exposure times to account for $160 \mu \mathrm{m}$ surface brightnesses at the optical radius, $R_{\mathrm{opt}}$ (the isophotal $B$-band surface brightness), which we were able to estimate thanks to Muñoz-Mateos et al. (2009b); thus for each "blue" wavelength $(70 \mu \mathrm{m}, 100 \mu \mathrm{m})$ there were three PACS scan repetitions for bright targets and six for faint ones. Because of the simultaneous

1 PACS has been developed by a consortium of institutes led by MPE (Germany) and including UVIE (Austria); KU Leuven, CSL, IMEC (Belgium); CEA, LAM (France); MPIA (Germany); INAFIFSI/OAA/OAP/OAT, LENS, SISSA (Italy); IAC (Spain). This development has been supported by the funding agencies BMVIT (Austria), ESA-PRODEX (Belgium), CEA/CNES (France), DLR (Germany), ASI/INAF (Italy), and CICYT/MCYT (Spain). 
Table 1. KINGFISH galaxy sample.

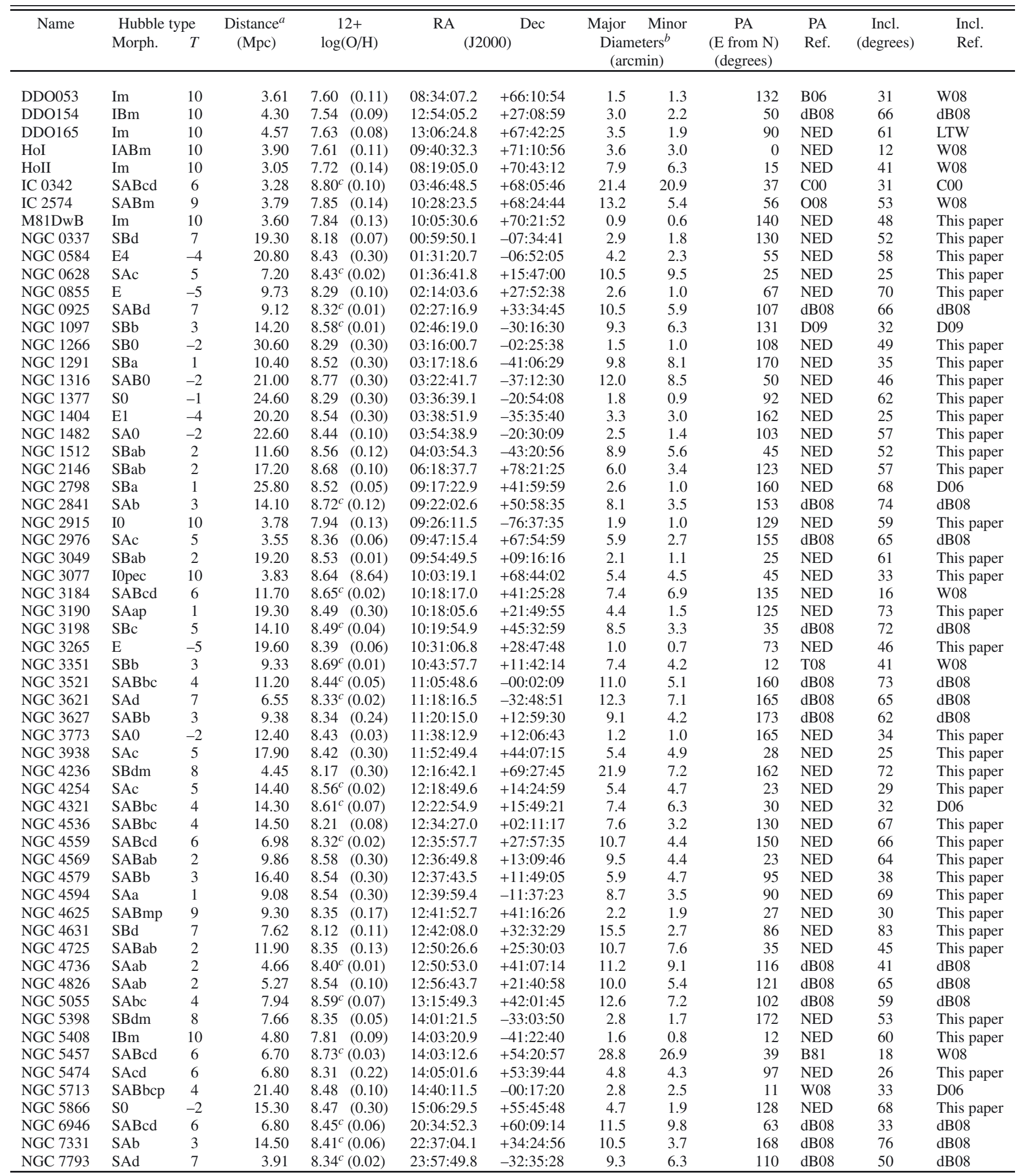

Notes. ${ }^{(a)}$ The method of distance determination is given by Kennicutt et al. (2011); ${ }^{(b)}$ Galaxy sizes are taken from NED = NASA/IPAC Extragalactic Database; ${ }^{(c)}$ Metallicity gradient from Moustakas et al. (2010), except for NGC 5457 (M 101) from Li et al. (2013) and IC 342 from Pilyugin et al. (2007). The latter has been adjusted to the mean slope ratio between Moustakas et al. (2010) and Pilyugin \& Thuan (2005), namely $\Delta \mathrm{O} / \mathrm{H}=-0.49$.

References. References for PAs and inclination angles: B06 = Begum et al. (2006); B81 = Bosma (1981); C00=Crosthwaite et al. (2000); D06 = Daigle et al. (2006); D09 = Davies et al. (2009); dB08 = de Blok et al. (2008); NED; O08 = Oh et al. (2008); T08= Tamburro et al. (2008); W08 = Walter et al. (2008). 
coverage at $160 \mu \mathrm{m}$, PACS $160 \mu \mathrm{m}$ has twice the number of scans as at the bluer wavelengths. SPIRE (Spectral and Photometric Imaging REceiver ${ }^{2}$, Griffin et al. 2010) mapping was performed with large-map mode at the nominal speed of $30^{\prime \prime} \mathrm{s}^{-1}$ with, as for PACS, two orthogonal scans. At the longest wavelengths, SPIRE maps are confusion limited (Nguyen et al. 2010), so for SPIRE we did not adopt two observing regimes. Because of the large angular extent of IC 342, one of the largest KINGFISH galaxies, we acquired PACS+SPIRE maps in parallel mode with two repetitions at slow speed $\left(20^{\prime \prime} \mathrm{s}^{-1}\right)^{3}$. We required both short PACS wavelengths, so used two repetitions in parallel mode. All maps were at least 1.5 times the optical size of the galaxy, with a minimum map size of $10^{\prime} \times 10^{\prime}$ for the sake of efficiency.

The Herschel interactive processing environment (HIPE, v.8; Ott 2010) was used for the image processing up to Level 1. Then for both PACS and SPIRE, images were converted from HIPE Level 1 to final maps with the scanamorphos algorithm (v. 16.9) (Roussel 2013). This method seemed to give the best estimate of faint diffuse low-surface brightness flux with PACS, since before projecting the data onto a spatial grid, it subtracts the brightness drifts caused by low-frequency flicker noise in the bolometers and the thermal drifts of the detectors and the telescope. After producing the final maps, both sets of images were corrected astrometrically to be aligned with the MIPS $24 \mu \mathrm{m}$ images (see below).

PACS calibrations correspond to the V7 responsivity calibration (global calibration V65). This has been accomplished by rescaling the original V6 fluxes obtained in the HIPE context with multiplicative factors of 1.0, 1.0152, 1.0288 at $70 \mu \mathrm{m}$, $100 \mu \mathrm{m}$, and $160 \mu \mathrm{m}$, respectively. Finally, to take into account an internal inconsistency of mappers external to HIPE (corrected in March, 2014), we multiplied the PACS $160 \mu \mathrm{m}$ flux by $0.925^{4}$.

SPIRE calibrations changed during the course of our analysis, so we multiplied the original (from HIPE v.8) fluxes by $0.9282(250 \mu \mathrm{m}), 0.9351(350 \mu \mathrm{m})$, and $0.9195(500 \mu \mathrm{m})$ in order to correct the images to the revised flux scale (see Griffin et al. 2013) $)^{5}$. These factors also take into account an internal discrepancy that introduced an error in the surface brightness units of the SPIRE maps (see the KINGFISH Data Release 3 document $^{6}$ ). Finally, to adjust to the latest HIPE 11 calibration, SPIRE fluxes were further multiplied by factors of $1.0321,1.0324$, and 1.0181, at $250 \mu \mathrm{m}, 350 \mu \mathrm{m}$, and $500 \mu \mathrm{m}$, respectively.

SPIRE images of six of the 61 galaxies were not acquired in the context of the KINGFISH program but rather by the Herschel

\footnotetext{
2 SPIRE has been developed by a consortium of institutes led by Cardiff University (UK) and including Univ. Lethbridge (Canada); NAOC (China); CEA, LAM (France); IFSI, Univ. Padua (Italy); IAC (Spain); Stockholm Observatory (Sweden); Imperial College London, RAL, UCL-MSSL, UKATC, Univ. Sussex (UK); and Caltech, JPL, NHSC, Univ. Colorado (USA). This development has been supported by national funding agencies: CSA (Canada); NAOC (China); CEA, CNES, CNRS (France); ASI (Italy); MCINN (Spain); SNSB (Sweden); STFC, UKSA (UK); and NASA (USA).

3 Despite its size, the Herschel images for NGC 5457 (M 101) were acquired in the same way as the smaller galaxies, not in parallel mode. 4 NGC 584 was observed much later than the other KINGFISH galaxies, and thus only the factor of 0.925 was applied to the $160 \mu \mathrm{m}$ flux for this galaxy.

5 For more information, please refer to the document posted at http://www.astro.princeton.edu/ draine/ Notes_re_KINGFISH_SPIRE_Photometry.pdf

$6 \mathrm{ftp}: / / \mathrm{hsa}$.esac.esa.int/URD_rep/KINGFISH-DR3/
}

Reference Survey (HRS, Boselli et al. 2010). However, these images have been reduced with the KINGFISH pipeline so as to be consistent with the remainder of the sample. Further details on data acquisition and reduction are given by Kennicutt et al. (2011).

\subsection{Spitzer IRAC and MIPS data}

We included in the SED analysis data from Spitzer, namely images acquired with IRAC (InfraRed Array Camera, Fazio et al. 2004), and with MIPS (Multiband Imaging Photometer, Rieke et al. 2004). Most of these were taken in context of the SINGS survey (Kennicutt et al. 2003), but many galaxies were also observed in the Local Volume Legacy (LVL, Dale et al. 2009), and we adopted that reduction when available. As mentioned above, four of the 61 KINGFISH galaxies are not in SINGS; for these we retrieved IRAC and MIPS data from the Spitzer archive, and reduced them using the LVL pipeline (Dale et al. 2009). A correction for non-linearities in the MIPS $70 \mu \mathrm{m}$ images was applied as described by Dale et al. (2009) and Gordon et al. (2011), and extended-source corrections were applied to the IRAC fluxes as described in Aniano et al. (2012).

\section{The radial brightness profiles}

Before extracting the elliptically averaged profiles, the images were processed to ensure that the SEDs would be reliable representations of the galaxies. Following Aniano et al. (2012), first the background light was estimated and subtracted by fitting tilted planes to the empty sky regions of each image. Bright sources and faint background galaxies were masked out a priori. The procedure was iterated in a clipping algorithm by successively minimizing the dispersion of the candidate background pixels around the best-fit plane, and upon convergence is subtracted from the image using the native pixel grid. Then, all images were convolved to a common point-spread function (PSF), in our case the lowest resolution, MIPS160, with a full width at half maximum (FWHM) of $38^{\prime \prime} .8$. This choice enables inclusion in the SED of all possible instruments (IRAC, MIPS, PACS, SPIRE) and is considered the "gold standard" by Aniano et al. (2012). Finally, the images were rebinned to a common pixel size, 18" (roughly half the FWHM of the MIPS160 beam), and astrometrically aligned. Care was taken to ensure that the final maps including all instruments covered at least 1.5 times the optical size of the galaxy. Further details of the comprehensive image analysis are given by Aniano et al. (2012). The width of the PSF (38'. 8 ) corresponds to $1.86 \mathrm{kpc}$ at the median KINGFISH sample distance of $9.9 \mathrm{Mpc}$.

\subsection{Extracting the profiles}

From these images, elliptically-averaged radial surface brightness profiles were extracted using the IRAF task ellipse. Centers, position angles (PAs), and ellipticities $(1-b / a$, where $a$ and $b$ are the major and minor axes of the galaxy) were kept fixed to the values reported in Table 1 . To circumvent possible slight (fraction of a pixel) misalignment despite the astrometric corrections described above, the profiles were extracted centered on the RA and Dec. coordinates as given in Table 1. The width (along the major axis) of each annulus is the same as the pixel size, $18^{\prime \prime}$, and the radial profile extraction extends in linear increments to at least 1.5 times the optical radius, $R_{\text {opt }}$. 

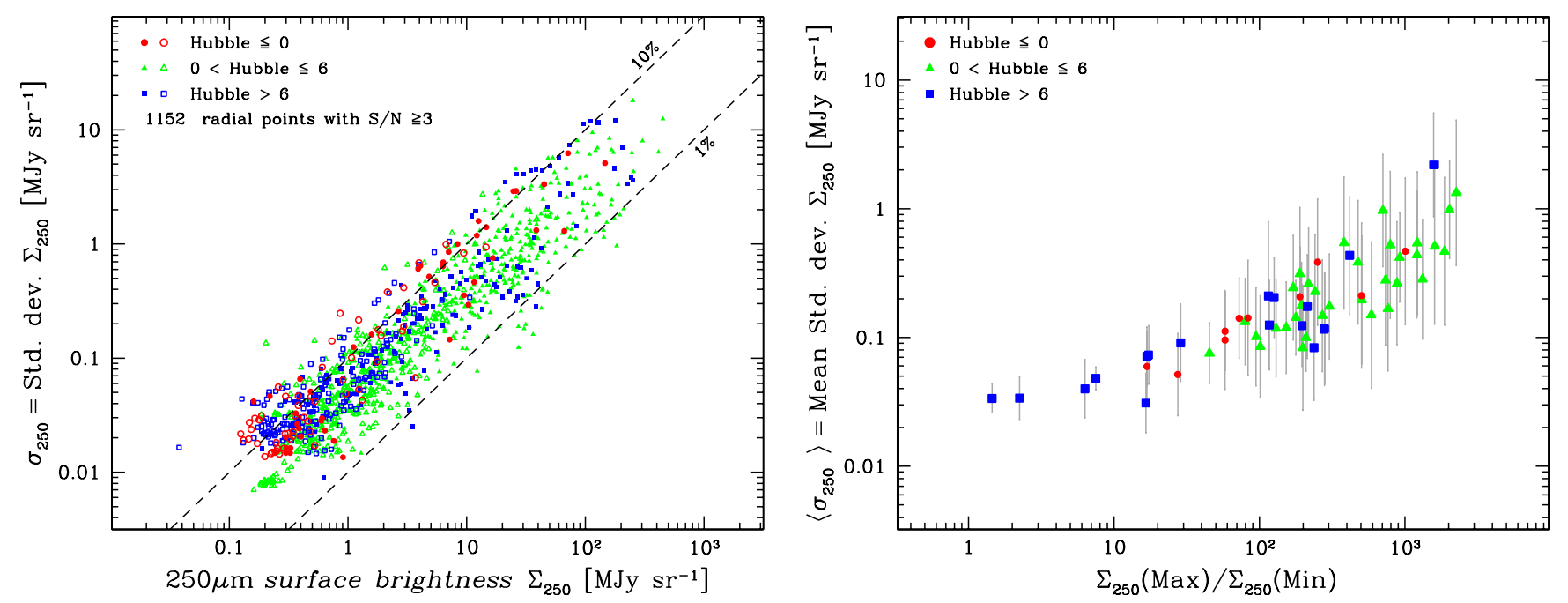

Fig. 1. Standard deviation $\sigma_{250}$ of the $250 \mu \mathrm{m}$ surface brightness within an annulus vs. $250 \mu \mathrm{m}$ surface brightness $\Sigma_{250}$ in the annulus (left panel), and the mean $\sigma_{250}$ over all the profiles in a galaxy vs. the ratio of maximum and minimum $\Sigma_{250}$ in the galaxy (right panel). Only radial points with $S / N \geq 3$ are shown. In the left panel, the dashed lines correspond to $10 \%$ variation (upper) and $1 \%$ variation (lower) relative to $\Sigma_{250}$. The error bars in the right panel reflect the standard deviation within a given profile. In both panels, points are plotted by Hubble type with (red) circles corresponding to early types $(T \leq 0)$, (green) triangles to spirals $(0<T \leq 6)$, and (blue) squares to late types $(T>6)$; in the left panel filled symbols correspond to $R / R_{\mathrm{opt}} \leq 0.8$ and open ones to larger radii.

As shown in Table 1, when possible, we adopted PAs and inclination angles $i$ determined kinematically. When these were not available, inclinations $i$ were calculated photometrically as a function of axial ratio according to Hubble (1926):

$\cos ^{2} i=\frac{(b / a)^{2}-q_{0}^{2}}{1-q_{0}^{2}}$.

For Hubble types $T<4$, we take $q_{0}=0.2$ for the intrinsic flattening of the galactic disk (Holmberg 1958), while Hubble types Sbc and later $(T \geq 4)$ were assumed to be intrinsically more flattened with $q_{0}=0.13$ (e.g., Giovanelli et al. 1994; Dale et al. 1997; Murphy et al. 2008). To avoid misrepresenting the elliptically averaged brightness profiles of highly inclined galaxies by including points too close to the projected minor axis, we applied a masking technique during ellipse fitting. For galaxies with inclination $i \geq 60^{\circ}$, we generated masks which removed from consideration in the elliptical averages a wedge-shaped subset of points around the minor axes. After several tests, the opening half-angle of the wedge was defined to be $30^{\circ}$ along the minor axis for $i=60^{\circ}$ and to vary linearly to a maximum of $80^{\circ}$ at the highest inclinations. Such a technique is particularly important for galaxies such as NGC 4631 and NGC 7331, both of which have $i>75^{\circ}$. Table 1 gives the parameters used for the ellipse extraction, together with the references for PAs and inclinations.

Uncertainties in the surface brightnesses as a function of radius were calculated as the quadrature sum of the variation along the elliptical isophotes and the calibration fractional uncertainties as given by Aniano et al. (2012). The latter are 0.083, 0.071, $0.221,0.167$ (i.e., $8.3 \%, 7.1 \%, 22.1 \%, 16.7 \%$ ) for the four IRAC channels, respectively, and 0.10 for MIPS, PACS, and SPIRE.

The MIPS 70 and MIPS 160 filters are not identical to the PACS 70 and PACS 160 filters, so that the two instruments will report different flux densities for the same nominal wavelength. For MBB spectra with $\beta=2$, and $T$ varying from 15 to $30 \mathrm{~K}$, the PACS/MIPS(70) flux ratio should vary from 1.059 to 0.965 , while the PACS/MIPS(160) ratio varies from 0.919 to 0.944 . For our sample the PACS/MIPS(70) mean flux ratio $=1.11 \pm 0.23$, and the mean flux ratio PACS/MIPS $(160)=0.90 \pm 0.19$. These mean values are roughly consistent with the estimated $10 \%$ calibration uncertainties for MIPS and PACS, but in individual cases the MIPS and PACS flux density ratios can differ from the expected ratio by more than this. Thus in order not to unduly confound the fitting procedure, the uncertainties for the MIPS and PACS data were taken to be 0.5 of the absolute value of the difference in fluxes between the two instruments; the uncertainties for PACS $100 \mu \mathrm{m}$ data were adjusted to be the mean of those at $70 \mu \mathrm{m}$ and $160 \mu \mathrm{m}$.

The uncertainties of the annular flux extractions were calculated as the error in the mean, namely the standard deviation $\sigma_{\lambda}$ of the distribution along the elliptical isophote circumference divided by the square root of the number of pixels in the circumference, $N_{\text {isophote }}$; this is then added in quadrature to the calibration uncertainties to obtain the total error. In the outer regions, the uncertainties are generally dominated by calibration errors because of the relatively large numbers of pixels. We have not considered the errors in the sky subtraction because of the complex method used to subtract background emission combined with the image degrading produced by convolution to the common MIPS $160 \mu \mathrm{m}$ resolution (Aniano et al. 2012). However, we have measured the noise of the sky subtraction of the original images by measuring the sky in 20 empty regions around the galaxies. The variation is in all cases much smaller than either the calibration uncertainties or the standard deviation of the elliptical isophotes.

\subsection{Variation within the radial profiles and dynamic range}

We know galaxies have structure (e.g., spiral arms, bars) not described by their radial profiles. Here we investigate the internal variation of the profiles for each galaxy and the dynamic range of their radial gradients by comparing the surface-brightness dispersion within each annulus to the ratio of the profile maximum and minimum brightness. For this analysis, we have chosen the brightness at $250 \mu \mathrm{m}$, in order to maximize the number of data points (see Sect. 5.2). Figure 1 shows the results of these comparisons on the convolved images. In the left panel, 

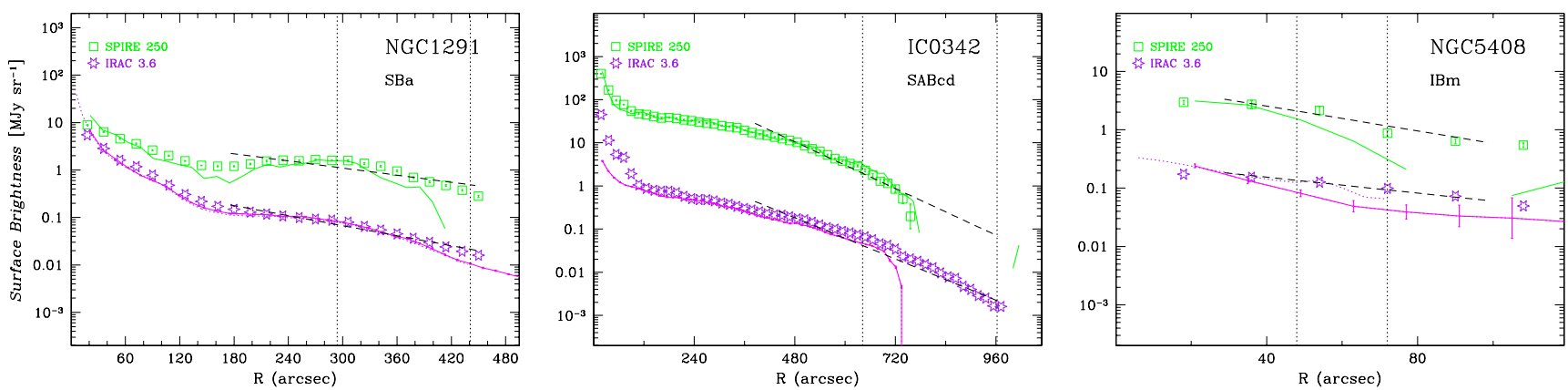

Fig. 2. Radial surface brightness profiles at IRAC $3.6 \mu \mathrm{m}$ and SPIRE $250 \mu \mathrm{m}$ shown in log-linear scale (cf., Fig. 7 with log-log) for NGC 1291 (left panel), IC 342 (middle), and NGC 5408 (right). Open (green) squares show the $250 \mu \mathrm{m}$ profiles and open (purple) stars $3.6 \mu \mathrm{m}$. The IRAC $3.6 \mu \mathrm{m}$ profiles from from Muñoz-Mateos (priv. comm.) correspond to solid (purple) curves, and the $250 \mu \mathrm{m}$ profiles extracted in a similar way (i.e., without the convolution by Aniano et al. 2012) as solid (green) curves. The dashed regression lines indicate the best-fit disk model fitted from $0.6 * R_{\mathrm{opt}}$; The vertical dotted lines indicate the optical radius, $R_{\mathrm{opt}}$, and $1.5 R_{\mathrm{opt}}$.

all radial points are shown with the standard deviation $\sigma_{250}$ of each annulus plotted against the surface brightness $\Sigma_{250}$ of that annulus ${ }^{7}$. Both $\Sigma_{250}$ and $\sigma_{250}$ vary by roughly 3 orders of magnitude over the galaxies in the KINGFISH sample, with the standard deviation roughly proportional to the surface brightness. Nevertheless, at a given surface brightness, $\sigma_{250}$ can vary by almost a factor of 10 , but at an amplitude between 10 and 100 times smaller than the surface brightness itself. Thus, the dynamic range of the profiles exceeds the internal variation by a factor of 10 or more.

This is perhaps better illustrated in the right panel of Fig. 1 where one point is given for each galaxy: the average variation $\left\langle\sigma_{250}\right\rangle$ is plotted against the dynamic range of the profile calculated as the ratio of maximum and minimum surface brightnesses within it. The error bars correspond to the mean standard deviation of each radial annulus in the galaxy. The internal dispersions of the annuli are much smaller than their dynamic range, except for four dwarf-irregular galaxies with rather flat profiles $\left(\Sigma_{250}(\operatorname{Max}) \Sigma_{250} /(\right.$ Min $) \lesssim 10$, e.g., DDO 154, DDO 165 with only one radial point, DDO 53, Holmberg II). In these cases, the average $\sigma_{250}$ is comparable to the lowest surface brightnesses, but 3 to 5 times fainter than the maximum $\Sigma_{250}$. Except for these extreme cases, the internal dispersion within each annulus is much smaller than the radial gradient, so that our analysis should reflect real radial trends, rather than just random noise introduced by internal variation within the rings.

\subsection{Radial distributions of dust as exponential disks}

Because dust is generally distributed in an exponential disk (e.g., Haas et al. 1998; Bianchi 2007; Muñoz-Mateos et al. 2009a), we have fit the radial profiles at $3.6 \mu \mathrm{m}$ and $250 \mu \mathrm{m}$ with a simple exponential $\Sigma=\Sigma_{0} \exp \left(-R / R_{\mathrm{d}}\right)$ starting from normalized radius $R / R_{\text {opt }} \geq 0.6$. These exponential fits are not intended as true bulge-disk decompositions, but rather as a characterization of the cool-dust distribution (at $250 \mu \mathrm{m}$ ) relative to the stars (traced by $3.6 \mu \mathrm{m}$ emission). Because we want to avoid contamination by the bulge, the fits consider only the external regions of the galaxy, with $R / R_{\mathrm{opt}} \geq 0.6$, an arbitrary value chosen because the contribution from the bulge is usually negligible at these radii, even in early-type spirals (e.g., Moriondo et al. 1998). $\mathrm{PAH}$ emission at $3.3 \mu \mathrm{m}$ in very dusty and active star-forming

\footnotetext{
7 These standard deviations $\sigma$ are not the same as the errors in the mean described in the previous section because they have not been divided by $\sqrt{N_{\text {isophote }}}$.
}

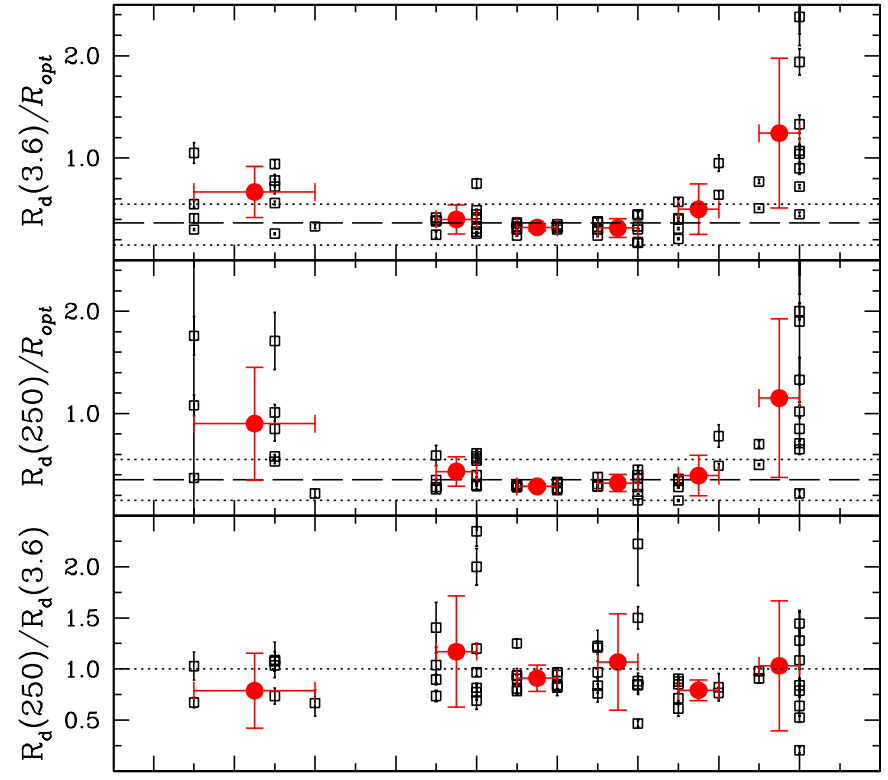

Fig. 3. Disk scalelengths normalized to $R_{\text {opt }}$ as a function of Hubble type $T$. The top panel shows the individual $3.6 \mu \mathrm{m}$ scalelengths $R_{\mathrm{d} 3.6}$, the middle the individual $250 \mu \mathrm{m}$ scalelengths $R_{\mathrm{d} 250}$, and the bottom their ratio. In each panel, the large circles with error bars report the means over a small range of Hubble types as indicated by the horizontal error bars; the vertical error bars are the standard deviations. The horizontal dashed lines in the top two panels show the mean scalelength obtained by averaging over spirals only $(1 \leq T \leq 5)$; the dotted lines show the range from 0.25 to 0.45 . In the bottom panel, the dotted line shows a scalelength ratio of unity.

galaxies could contribute significantly to the $3.6 \mu \mathrm{m}$ flux, which may produce a slightly tighter correlation with $250 \mu \mathrm{m}$ emission than would be expected from a comparison of pure stellar emission and dust (e.g., Zibetti \& Groves 2011); however, this is not expected to affect our conclusions. Representative fits ${ }^{8}$ are illustrated graphically in Fig. 2.

The resulting normalized disk scalelengths ${ }^{9} R_{\mathrm{d}} / R_{\text {opt }}$ are shown in Fig. 3 where the top and middle panels show the

\footnotetext{
8 The entire suite of exponential-fit plots is available in Appendix B.

9 Because some dwarf galaxies (DDO 165, Holmberg I, M 81dwB) and one elliptical (NGC 1404) have only one point in the multi-wavelength SED radial profiles, these galaxies are not considered in the exponential fit analysis.
} 

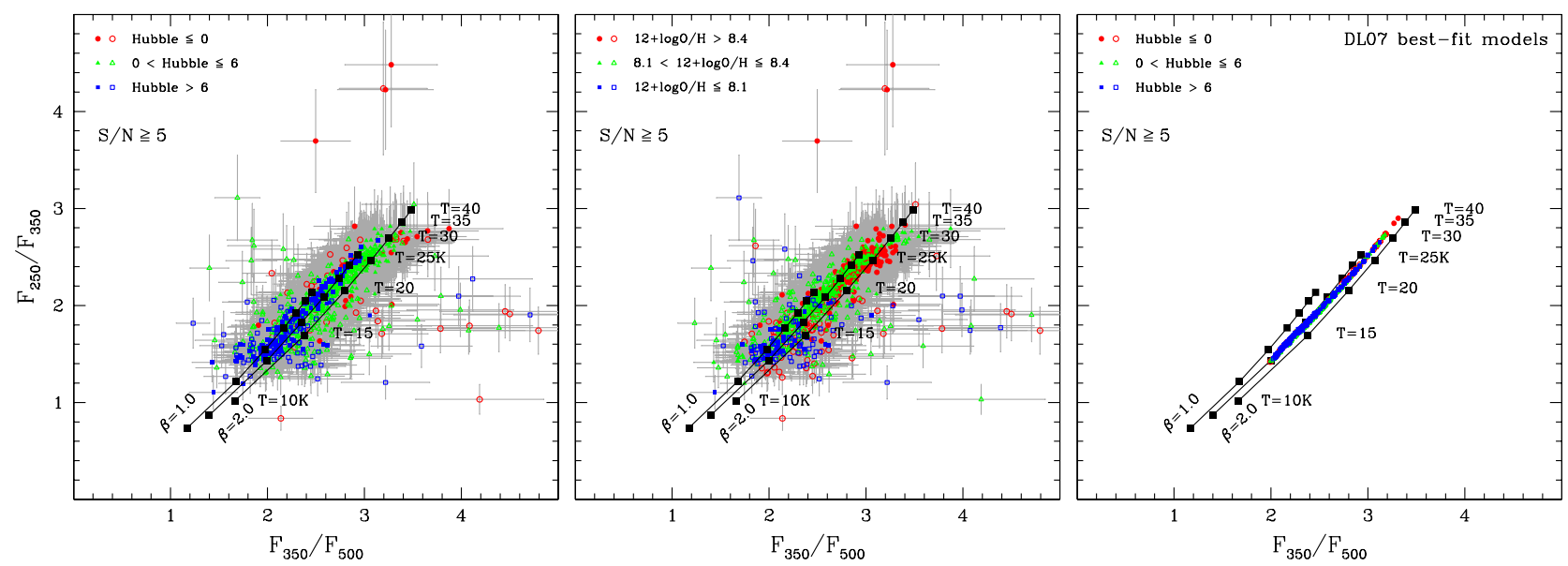

Fig. 4. SPIRE flux ratios of the radial profiles with $S / N \geq 5$. In the left and right panels, points are plotted by Hubble type with (red) circles corresponding to early types $(T \leq 0)$, (green) triangles to spirals $(0<T \leq 0)$, and (blue) squares to late types $(T>6)$. In the middle panel, points are distinguished by $\mathrm{O} / \mathrm{H}$ with (red) circles showing $12+\log (\mathrm{O} / \mathrm{H})>8.4$, (green) triangles $8.0<12+\log (\mathrm{O} / \mathrm{H}) \leq 8.4$, and (blue) squares $12+\log (\mathrm{O} / \mathrm{H}) \leq 8.1$. Filled symbols correspond to positions with normalized (to optical radius $R_{\mathrm{opt}}$ ) radii within $R / R_{\mathrm{opt}} \leq 0.8$, and open symbols to larger radii. The right panel shows DL07 best-fit models only. In all panels, MBB fits are shown with three values of emissivity index $\beta$ : from left to right $\beta=1.0, \beta=1.5, \beta=2.0$, respectively. Temperatures are also labeled from $T_{\text {dust }}=10 \mathrm{~K}$ to $T_{\text {dust }}=40 \mathrm{~K}$.

scalelengths of the $3.6 \mu \mathrm{m}$ and $250 \mu \mathrm{m}$ profiles, and the bottom panel their ratio. The mean values in the two top panels, shown as horizontal dotted lines, correspond to the means over only spirals $(1 \leq T \leq 5)$, and are similar for both wavelengths: $R_{\mathrm{d}} / R_{\mathrm{opt}}=0.37 \pm 0.11$ for $3.6 \mu \mathrm{m}$ and $R_{\mathrm{d}} / R_{\mathrm{opt}}=0.35 \pm 0.12$ for $250 \mu \mathrm{m}$. These normalized disk scalelength are larger than, although comparable to, those found for stars in spiral galaxies from detailed two-dimensional bulge-disk decompositions (0.25-0.30, e.g., Giovanelli et al. 1994; Moriondo et al. 1998; Hunt et al. 2004); they are also slightly larger than, although within the uncertainties, the median value, 0.29 , of the dust scalelengths in SINGS galaxies found by Muñoz-Mateos et al. (2009a). The slight increase in the disk scalelengths with respect to previous work could be due to the method of sky subtraction in the convolved images we are using here; if less sky were subtracted, disks would be shallower with larger scalelengths. Nevertheless, the similar processing of both the $3.6 \mu \mathrm{m}$ and $250 \mu \mathrm{m}$ images should obviate potential biases that could affect our conclusions.

The salient point is that the exponential distributions of the cool dust and the stars are similar, as can be seen in the bottom panel of Fig. 3. The mean of the ratio of the $250 \mu \mathrm{m}$ and $3.6 \mu \mathrm{m}$ scalelengths is $0.96 \pm 0.36$; the median of the ratio is even smaller, 0.88 , with quartile spreads of $\sim 0.2$. There is a tendency for both early-type galaxies $(T<0,10$ galaxies $)$ and very late-type galaxies ( $T \gtrsim 9,10$ galaxies) to have larger exponential scalelengths, both in the dust and in the stars. Three spiral galaxies, NGC 5457 (1.5), NGC 5474 (2.2), NGC 4826 (2.3), have scalelength ratios $\gtrsim 1.5$, so in some cases, spiral dust disks are more extended than their stellar ones.

In general, however, our results are roughly consistent with the finding of Muñoz-Mateos et al. (2009a) that the dust distribution can be more extended than the stars by $\sim 10 \%$ at most. On the other hand, radiative transfer models of dust and stars in edge-on spirals suggest that dust can be significantly more extended (Xilouris et al. 1999; Bianchi 2007; Holwerda et al. 2012a), although this is not a general rule (e.g., Bianchi \& Xilouris 2011). Analyses of the radial trends of dust extinction also imply that the dust distribution extends beyond the stellar one (e.g., Holwerda et al. 2005). With our simple analysis, we find that both dust and stars are distributed in an exponential decline with similar scalelengths; the cool dust in most KINGFISH galaxies traced by $250 \mu \mathrm{m}$ emission has a similar distribution to the stellar one. However, the stellar populations traced by IRAC $3.6 \mu \mathrm{m}$ emission include both main sequence and red giant stars, but some studies have shown that dust emission is more closely related to somewhat younger populations as traced by the optical $B$ band (e.g., Alton et al. 1998). Comparison of radial gradients of SFR, stellar age, or SFH would be necessary to link our result with scenarios for insideout disk growth (e.g., Williams et al. 2009).

\section{Radial far-infrared colors}

Before pursuing the relation between the physical parameters driving the DL07 models, and their connection with MBB fits, we present here color-color diagrams for the $\sim 900$ SEDs from the radial profile data independently of their position. Figure 4 shows the SPIRE flux ratios, $F_{250} / F_{350}$ vs. $F_{350} / F_{500}$. The left and middle panels show the data, coded by either Hubble type, $\mathrm{T}$, or oxygen abundance, $12+\log (\mathrm{O} / \mathrm{H})$, and the right panel shows the best-fit DL07 models described in Sect. 5.3. All panels show only data with $S / N \geq 5$; we have used this relatively high $\mathrm{S} / \mathrm{N}$ threshold because we are examining colors and want to ensure their reliability. Single-temperature fixed-emissivity MBB models are also illustrated with three values of emissivity index $\beta$; the MBB models have been integrated over the SPIRE response function for comparison with the observed SPIRE fluxes. Dust emitting at a single temperature with a single $\beta$ would occupy a single point on such a plot.

Figure 4 illustrates that the data have a wide range of apparent dust temperatures and generally fall between $\beta=1$ and $\beta=2$, as expected from previous work (e.g., Boselli et al. 2012). Nevertheless, even at this significant $S / N \geq 5$, there are clear variations. Figure A.1 plots SPIRE colors against galactocentric distance and shows that most of the data that might indicate a potential $500 \mu \mathrm{m}$ excess (e.g., $F_{350} / F_{500} \lesssim 1.5$ ) are in the external regions of the galaxies $\left(R / R_{\text {opt }}>0.8\right)$. However, not all galaxies with a possible excess are either low metallicity $(12+\log (\mathrm{O} / \mathrm{H}) \leq 8.1)$ or late type $(T>6)$. The search for long-wavelength excesses will be discussed in Sect. 7.5. 

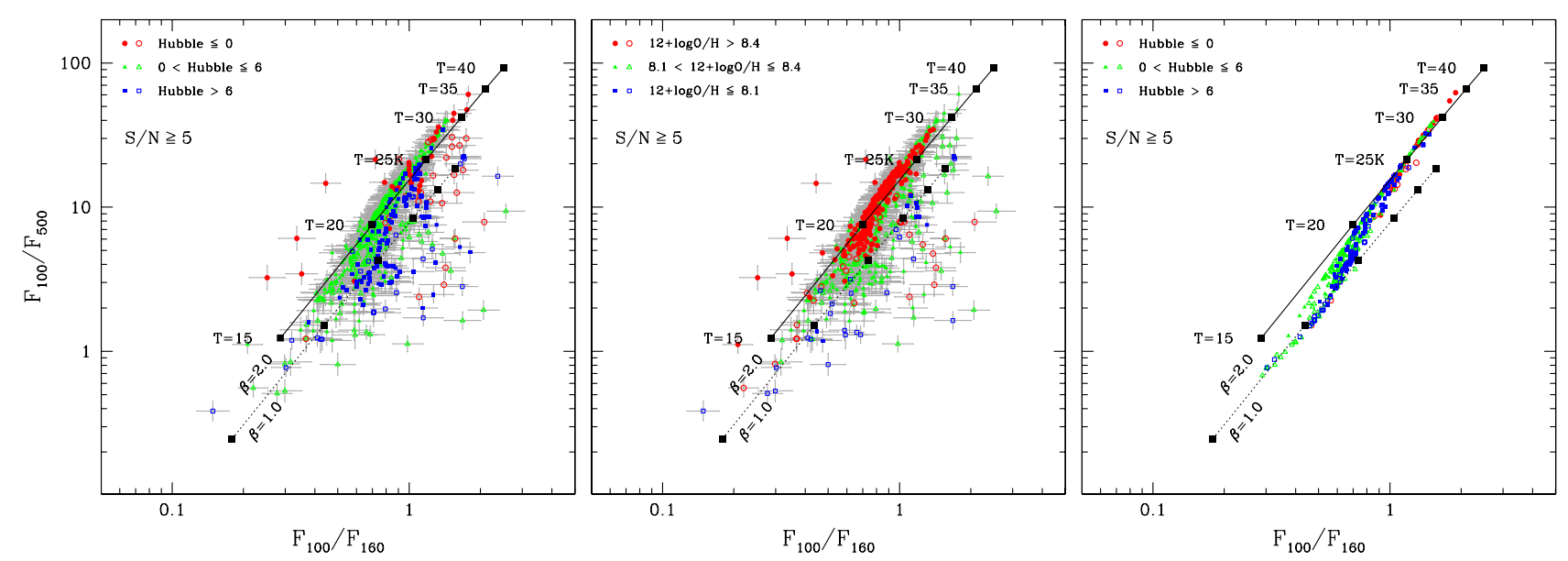

Fig. 5. PACS $(100 \mu \mathrm{m}, 160 \mu \mathrm{m})$ and SPIRE $500 \mu \mathrm{m}$ flux ratios of the radial profiles with $S / N \geq 5$. As in Fig. 4 , in the left and right panels, points are plotted by Hubble type with (red) circles corresponding to early types $(T \leq 0)$, (green) triangles to spirals $(0<T \leq 0)$, and (blue) squares to late types $(T>6)$. In the middle panel, points are distinguished by $\mathrm{O} / \mathrm{H}$ with (red) circles showing $12+\log (\mathrm{O} / \mathrm{H})>8.4$, $(\mathrm{green})$ triangles $8.0<12+\log (\mathrm{O} / \mathrm{H}) \leq 8.4$, and (blue) squares $12+\log (\mathrm{O} / \mathrm{H}) \leq 8.1$. Filled symbols correspond to positions with normalized (to optical radius $R_{\mathrm{opt}}$ ) radii within $R / R_{\text {opt }} \leq 0.8$, and open symbols to larger radii. The right panel shows DL07 best-fit models only. In all panels, MBB fits are shown with two values of emissivity index $\beta: \beta=1.0$ (dotted line) and $\beta=2.0$ (solid line). Temperatures are also labeled from $T_{\text {dust }}=15 \mathrm{~K}$ to $T_{\text {dust }}=40 \mathrm{~K}$.

Figure 4 also demonstrates the unexpected result that SPIRE colors are not necessarily indicative of the average emissivity index of the dust. The range in colors spanned by the best-fit DL07 models (see Sect. 5.3) is narrower than observed, and the best-fit DL07 colors in the right panel do not fall on $\beta=2$, as would be expected given the mean DL07 value of $\beta$ of 2.08 (Draine \& Li 2007; Bianchi 2013). The apparent emissivity index of the DL07 models in this wavelength range is flatter, $\beta \sim 1.5$ with a maximum value toward warmer temperatures of $\beta \sim 1.7$ at SPIRE wavelengths. This behavior depends on the intrinsic grain properties of dust in the DL07 models at SPIRE wavelengths. Although the mean emissivity of the DL07 models over the whole PACS and SPIRE range can be globally described by a power law with $\beta \sim 2.1$, the model emissivity at $\lambda \geq 250 \mu \mathrm{m}$ is better described by a broken power law, with a slightly steeper $\beta$ for the $250 / 350$ ratio than for the $350 / 500$ ratio ( $\beta$ decreasing from 2.2 to 2.0 ). These intrinsic properties of the dust model, leading to a $350 \mu \mathrm{m}$ emissivity $4 \%$ smaller than predicted by the $\beta=2.1$ power-law fit, are responsible for the colors of the DL07 best-fit models. The reason for the change in slope at the SPIRE wavelengths is due mostly to modifications in the optical properties of astronomical silicates, which were made by Li \& Draine (2001) to provide a better match between the model and the FIRAS high-latitude Galaxy spectrum. These results imply that IR colors over a small wavelength range should not be used to infer intrinsic dust properties.

PACS+SPIRE colors are given in Fig. 5 where we plot PACS/SPIRE $F_{100} / F_{500}$ vs. PACS $F_{100} / F_{160}$ (see also Cortese et al. 2014). As in Fig. 4, the left panel shows the data coded by Hubble type, the middle by $12+\log (\mathrm{O} / \mathrm{H})$, and the right panel shows the best-fit DL07 models (see Sect. 5.3). Singletemperature fixed-emissivity MBB models are illustrated here with two values of emissivity index $\beta$; as before, the MBB values have been integrated over the PACS and SPIRE response functions to be consistent with the scale of the PACS+SPIRE observed flux calibrations. Again, dust emitting at a single temperature with a given emissivity index $\beta$ would appear as a single point.

Figure 5 shows that for a given PACS color (horizontal axis) $F_{100} / F_{500}$ (vertical) can slightly exceed the ratios expected from a single-temperature MBB. In addition, the same $F_{100} / F_{500}$ color can also fall well below expectations, implying perhaps a $500 \mu \mathrm{m}$ excess. In any case, there is significant variation, and these trends will be discussed further in Sect. 7.5.

The best-fit DL07 models given in the right panel of Fig. 5 and described in Sect. 5.3 change apparent emissivity index as a function of $T_{\text {dust }}$. For low temperatures ( $T_{\text {dust }} \leqslant 25 \mathrm{~K}$ ), $\beta$ is lower than the nominal value of $\sim 2$, reaching an extreme of $\beta \sim 1$ for $T_{\text {dust }} \lesssim 20 \mathrm{~K}$. At higher temperatures, the best-fit DL07 models fall along the $\beta=2$ MBB fits, in accordance with the mean emissivity value. These simple color-color plots imply that fixedemissivity models can well approximate dust emission that is well fit by low values of $\beta$. We discuss possible reasons why in Sect. 7.

The FIR colors of galaxies vary with position, typically as a signature of radial temperature gradients. Such trends are illustrated in Fig. 6 where we show the SPIRE flux ratios of individual galaxies with more than 30 radial data points: IC 342, IC 2574, NGC 4236, NGC 4631, NGC 5055, NGC 5457. Observed SPIRE flux ratios correspond to cooler temperatures (occurring in the outer regions of the galaxies and apparently flatter emissivity $\beta \sim 1$ ). The DL07 best-fit models in Fig. 6 reproduce the SPIRE colors to within the uncertainties, although there may be systematic variations. We will examine these in more detail in Sect. 7.5.

\section{Modeling the radial dust SEDs}

Herschel has enabled the analysis of the cool dust in galaxies in a detail that was not previously possible. Although much work has focused on simple MBB fits of the dust SED, the application of more physically motivated dust models such as those of Draine \& Li (2007) can give greater physical insight. Here we combine both approaches and fit the SED of each annular region in each galaxy with three sets of models: the DL07 templates and MBB fits with variable (MBBV) and fixed (MBBF) emissivity index $\beta$. In addition, to better comprehend the relation between the ISRF and the apparent properties of dust grains that emerge from MBB fits, we fitted the best-fit DL07 models themselves with the two kinds of MBB fits. Thus, in total, we will analyze five sets of 

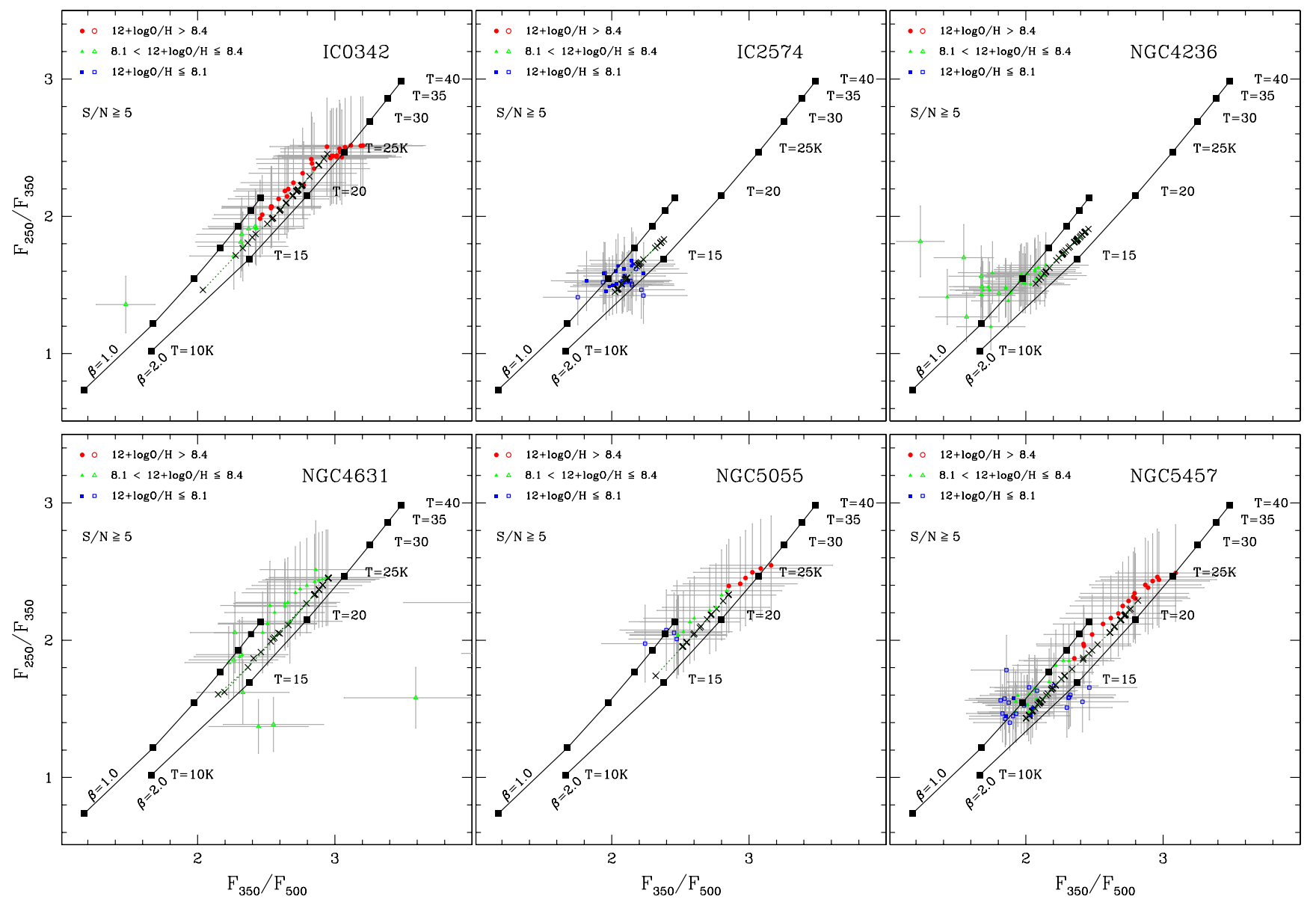

Fig. 6. SPIRE colors of individual galaxies with more than 30 radial data points. Data are distinguished by $\mathrm{O} / \mathrm{H}$ with (red) circles showing $12+\log (\mathrm{O} / \mathrm{H})>8.4$, (green) triangles $8.0<12+\log (\mathrm{O} / \mathrm{H}) \leq 8.4$, and (blue) squares $12+\log (\mathrm{O} / \mathrm{H}) \leq 8.1$. Filled symbols correspond to positions with normalized (to optical radius $R_{\mathrm{opt}}$ ) radii within $R / R_{\mathrm{opt}} \leq 0.8$, and open symbols to larger radii. DL07 best-fit models for the data are shown with $\times$, connected by a dotted line. In all panels, MBB fits are given for two values of emissivity index $\beta$ : $\beta=1.0$ (dotted line) and $\beta=2.0$ (solid line). Temperatures are also labeled from $T_{\text {dust }}=15 \mathrm{~K}$ to $T_{\text {dust }}=40 \mathrm{~K}$.

fits: three sets of model fits to data (MBBV, MBBF, DL07) and two sets of fits (MBBV, MBBF) to the best-fit DL07 models. The profile extraction gives 1166 radial data points, although we apply $\mathrm{S} / \mathrm{N}$ thresholds (see below), so in the analysis only a subset of these is considered ( $\sim 800$ data points).

\subsection{Modified blackbody fitting}

The SED of dust grains in local thermal equilibrium can be represented by a MBB:

$$
F_{v}=B\left(T_{\text {dust }}, v\right) \Omega\left(1-\mathrm{e}^{-\tau_{v}}\right) \approx B\left(T_{\text {dust }}, v\right) \Omega \tau_{v}
$$

where $F_{v}$ is the observed monochromatic flux, $B\left(T_{\text {dust }}, v\right)$ is the Planck function, $\Omega$ is the solid angle of the observing beam, and $\tau_{v}$ is the dust opacity. The approximation of $F_{v} \propto$ $B\left(T_{\text {dust }}, v\right) \tau_{v}$ holds in an optically thin regime which is what we will assume for the cool dust component of the KINGFISH galaxies. The opacity $\tau_{v}$ is directly proportional to the mass attenuation coefficient (alternatively emissivity or the grain absorption cross-section per unit mass):

$\tau_{v}=\Sigma_{\text {dust }} \kappa_{v}=\Sigma_{\text {dust }} \kappa_{0}\left(\frac{v}{v^{0}}\right)^{\beta}$

where $\Sigma_{\text {dust }}$ is the mass surface density of the dust and $\kappa_{v}$ is assumed to have a power-law dependence $\propto \nu^{\beta}$ (Hildebrand 1983), normalized to the frequency $v_{0}$ corresponding to $250 \mu \mathrm{m}$. For the DL07 Milky Way dust models (Draine \& Li 2007) ${ }^{10}$, $\kappa_{0}(250 \mu \mathrm{m})=0.40 \mathrm{~m}^{2} \mathrm{~kg}^{-1}$ and $\kappa_{0}(160 \mu \mathrm{m})=1.00 \mathrm{~m}^{2} \mathrm{~kg}^{-1}$. Hence, for optically thin dust, we can write:

$$
F_{v}=\Sigma_{d} B\left(T_{\text {dust }}, v\right) \Omega \kappa_{0}\left(\frac{v}{v^{0}}\right)^{\beta} .
$$

Such a description is not generally realistic because of temperature mixing along the line of sight (LOS) and because of the distribution of dust sizes, densities, and compositions in the general grain population. Nevertheless, despite its limitations, the MBB has been shown to provide a relatively good approximation of observed cool dust SEDs, at least for dust-mass determinations (e.g., Bianchi 2013).

The dust emissivity index, $\beta$, depends on the physical properties and chemical composition of the grains, and may also depend on environment and temperature (Mennella et al. 1995, 1998; Stepnik et al. 2003; Paradis et al. 2009; Coupeaud et al. 2011). For wavelengths $\lambda \gtrsim 100 \mu \mathrm{m}$, typical dust compositions with carbonaceous and silicate grains in diffuse high-latitude clouds have $\beta \approx 2$ ( $\mathrm{Li} \&$ Draine 2001; Draine \& Li 2007). Detailed observations of the Milky Way show that $\beta \sim 1.5-1.8$ in the Galactic plane and

${ }^{10}$ We will not be using $\kappa_{0}$ in this paper because we are not calculating dust masses, but give them here for completeness. 
the diffuse halo (Planck Collaboration XXIV 2011; Planck Collaboration Int. XIV 2014), but can be as high as $\beta \sim 2.8$ in Galactic cold clumps (Planck Collaboration XXII 2011). Global $\beta$ values from 1 to 2 are found in external galaxies (Boselli et al. 2012; Dale et al. 2012; Auld et al. 2013), and spatially resolved studies show a similar spread of $\beta$ even within a galaxy (Smith et al. 2012; Galametz et al. 2012; Kirkpatrick et al. 2014). Some galaxies tend to have systematically lower values of $\beta \sim 1.5-1.7$ (e.g., the SMC, LMC, M 33: Galliano et al. 2011; Planck Collaboration XVII 2011; Tabatabaei et al. 2014, respectively). These variations have been attributed to different metallicity or dust heating, but such causes are currently difficult to prove.

Studies of the Milky Way also show that the spectrum of the dust emission tends to flatten toward longer wavelengths so that $\beta$ in the FIR $(\lambda \lesssim 850 \mu \mathrm{m})$ is $\sim 0.2$ larger than in the submm $(\lambda \gtrsim 850 \mu \mathrm{m})$ regime (Planck Collaboration Int. XIV 2014). Observationally, there is apparently also a temperature dependence; higher (steeper) $\beta$ values tend to be associated with cooler dust temperatures, and lower (flatter) ones with warmer dust (Pollack et al. 1994; Paradis et al. 2009). Some laboratory experiments show similar results for the flattening of $\beta$ at long wavelengths and at high temperatures (e.g., Mennella et al. 1995; Agladze et al. 1996; Mennella et al. 1998; Coupeaud et al. 2011),

Despite these seemingly consistent results for the behavior of dust temperature $T_{\text {dust }}$ and emissivity index $\beta$, they have been challenged by various groups. LOS temperature mixing and measurement noise have been proposed as the causes of correlated variations of temperature and $\beta$ in sightlines in the Galaxy (Shetty et al. 2009a,b; Juvela \& Ysard 2012; Juvela et al. 2013). Such correlations, namely that large $\beta$ (steep slopes) tends to be associated with cool $T_{\text {dust }}$, emerge because equivalently good MBB fits (as measured by $\chi_{v}^{2}$ ) can give different, but related, values of $\beta$ and $T_{\text {dust }}$. Such degeneracy between $\beta$ and $T_{\text {dust }}$ in $\chi^{2}$ space is not unexpected when curve-fitting algorithms are used to simultaneously fit $T_{\text {dust }}$ and $\beta$, because the derivatives of the MBB function with respect to these parameters are correlated. Thus parameter estimation has become another topic of debate (e.g., Kelly et al. 2012).

In any case, results all indicate that the fitted dust emissivities inferred from a MBB fit are not straightforward to interpret. This is because both $T_{\text {dust }}$ and $\beta$ are luminosity-weighted apparent values that may not reflect intrinsic grain properties. Moreover, wavelength coverage, fitting technique, and data quality all influence the outcome of MBB fitting, meaning that results must be carefully assessed a posteriori.

\subsection{MBB fits of the radial-profile SED data}

Other papers have relied on more sophisticated methods for MBB fitting, including multiple temperature components (e.g., Kirkpatrick et al. 2014; Tabatabaei et al. 2014), additional long-wavelength coverage (Galametz et al. 2014), and broken power-law emissivities (Gordon et al. 2014). Here we adopt the simplest approach of a single temperature $T_{\text {dust }}$ and a single emissivity $\beta$. As mentioned above, we pursue MBBV fits with variable $\beta$ and MBBF fits where we fix $\beta=2$.

To be considered in the SED to be fit, each radial data point was required to have signal-to-noise ratio $(\mathrm{S} / \mathrm{N}) \geq 3$. Because $70 \mu \mathrm{m}$ emission may be contaminated by non-equilibrium emission from stochastically-heated grains (e.g., Draine \& Li 2001; Li \& Draine 2001; Compiègne et al. 2011), the fit did not include the MIPS or PACS $70 \mu \mathrm{m}$ (or any shorter wavelength) points. We however required the fit to not exceed the average $70 \mu \mathrm{m}$ flux plus its $1 \sigma$ uncertainty. The SPIRE $500 \mu \mathrm{m}$ data point was excluded from the fit in order to examine long-wavelength model predictions. Thus, the best-case SED would have 5 data points (2 PACS, 1 MIPS, 2 SPIRE). By not constraining the MBB at long wavelengths, we enable a potential search for a submm excess (e.g., Planck Collaboration XVII 2011; Galliano et al. 2011; Kirkpatrick et al. 2013; Galametz et al. 2014), as discussed in Sect. 7.6.

Because three parameters (normalization, $T_{\text {dust }}, \beta$ ) are needed for the MBBV fits, as recommended by Shetty et al. (2009b), we did not fit any profiles with fewer than $4(S / N \geq 3)$ data points. Photometric color corrections were applied to the MBB models before fitting, rather than applied directly to the data ${ }^{11}$. These take into account the Herschel color-dependent beam sizes (PACS, SPIRE) and also the observed spectral distribution of the source across the instrumental band-passes (IRAC, MIPS, PACS, SPIRE). Except for very low or very high temperatures, these corrections are usually on the order of a few percent.

The best-fit parameters were determined by minimizing reduced $\chi_{v}^{2}$. Traditional curve fitting uses $\chi^{2}$ gradients in parameter space to find the minimum, but the partial derivatives of temperature and emissivity index are correlated because of the mathematical form of the MBB function. Thus, such a procedure could induce potential correlations even when none are present in the data. Therefore, to ameliorate as far as possible any spurious correlation between $T_{\text {dust }}$ and $\beta$, we adopted a two-pass grid method of stepping through possible values of $\beta(0.5 \leq \beta \leq 3)$ and $T_{\text {dust }}$ $\left(5 \mathrm{~K} \leq T_{\text {dust }} \leq 45 \mathrm{~K}\right)$. Parameter uncertainties were calculated by assuming that the Hessian matrix is diagonal, and performing analytically second-order differentiation of the expression for $\chi^{2}$ with respect to $T_{\text {dust }}$ and $\beta$. Since the two parameters $T_{\text {dust }}$ and $\beta$ are correlated (making the Hessian not diagonal), the uncertainties will be underestimated.

We adopted the same algorithm for MBBF fits, except for fixing $\beta=2$. Again, a $S / N \geq 3$ was required for each radial data point. In the MBBF case, there is one fewer parameter to fit, and reduced $\chi_{v}^{2}$ was calculated accordingly. The fits are in any case rather under-constrained.

The $\mathrm{S} / \mathrm{N}$ requirements result in 920 radial data points with sufficient SPIRE S/N at all three wavelengths, and 766 with sufficient PACS S/N at both $100 \mu \mathrm{m}$ and $160 \mu \mathrm{m}$ (817 profile points have PACS $160 \mu \mathrm{m} \geq 3$ ). In the following analysis, only MBBV and MBBF fits with reduced $\chi_{v}^{2} \leq 2$ are considered. This, together with the $\mathrm{S} / \mathrm{N}$ requirement for individual surface brightness points $(S / N \geq 3)$, results in 818 SED radial profile MBBV fits and $827 \mathrm{MBBF}$ fits (of a total of 1166 radial data SEDs for 61 galaxies). Given the similar number of good (low $\chi_{v}^{2}$ ) MBBF fits, we conclude that the MBBF (fixed-emissivity) fits are as able as the MBBV ones to approximate the true data SEDs.

\subsection{Physically realistic dust models}

To better approximate the true physical conditions of the dust grains, we also fitted the radial profile data SEDs with DL07 models. These models assume that dust is composed of a mixture of silicates, graphites, and polycyclic aromatic hydrocarbons (PAHs), with size distributions (Weingartner \& Draine 2001) that reproduce the wavelength-dependent extinction observed in the Milky Way. The dust is heated by an ISRF with a Milky-Way like spectrum (Mathis et al. 1983), with a distribution of starlight intensities.

\footnotetext{
11 See the online documentation at http://herschel. esac. esa.int/twiki/bin/view/Public
} 
The starlight intensity distribution is described as a truncated power law with four parameters: $U_{\min }$, the minimum ISRF level; $U_{\max }$, the upper limit of ISRF level; $\alpha$, the power-law index of the ISRF distribution; and $\gamma$, the fraction of the dust mass exposed to starlight with intensities greater than $U_{\min }$. The fraction of the dust mass exposed to a distribution of starlight intensities $U$ is defined as

$$
\frac{\mathrm{d} M_{\text {dust }}}{\mathrm{d} U}=(1-\gamma) M_{\text {dust }} \delta\left(U-U_{\text {min }}\right)+\gamma M_{\text {dust }} \frac{\alpha-1}{\left(U_{\max }^{1-\alpha}-U_{\min }^{1-\alpha}\right)} U^{-\alpha}
$$

where the power-law index, $\alpha \neq 1$, and $M_{\text {dust }}$ is the total dust mass. Remaining parameters in the DL07 fits include $q_{\mathrm{PAH}}$, the fraction of dust mass composed of PAHs, and $f_{\mathrm{PDR}}$, the fraction of dust emission radiated by grains exposed to intense levels of ISRF $\left(U>10^{2}\right)$. More details and recipes for calculating $q_{\mathrm{PAH}}$ and $f_{\mathrm{PDR}}$ are given by Draine \& Li (2007).

The functional form of the DL07 models is similar to the power-law distribution formulated by Dale et al. (2001) and Dale $\&$ Helou (2002), but with an added delta-function component of dust heated by $U_{\min }$. This is the component that dominates the dust mass, representing the dust mass in the general diffuse ISM. The shape of the dust emission spectrum is governed by $q_{\mathrm{PAH}}, U_{\mathrm{min}}$, and $\gamma ; q_{\mathrm{PAH}}$ and $\gamma$ generally determine the shortwavelength mid-infrared (MIR) region $(\$ 70 \mu \mathrm{m})$ while $U_{\min }$ the long-wavelength FIR regime $(\gtrsim 70 \mu \mathrm{m})$. A useful parameter to link total dust mass $M_{\text {dust }}$ with the luminosity of the dust emission is $\langle U\rangle$, or the mean starlight intensity; it is uniquely defined by $U_{\min }, U_{\max }$, and $\gamma$. As shown by Draine \& Li (2007), $\langle U\rangle$ is inversely proportional to the mass-to-light ratio of the dust. Like the MBB fits to the data, the DL07 models were integrated over the instrumental response functions for comparison with observed fluxes.

There are 13 data points in the DL07 fits (4 IRAC, 3 each MIPS, PACS, SPIRE); hence the fits are somewhat over-constrained, although there are several free parameters including the ISRF starlight intensity determined by the IRAC fluxes. However, the long-wavelength regime of the dust SEDs is only part of the entire spectrum fit by the DL07 models. Consequently, the quality of the fit depends on the dust optical properties and albedo assumed for the DL07 grain populations. The MBB fits are required only to fit the $100 \mu \mathrm{m} \leq \lambda \leq 350 \mu \mathrm{m}$ SED, and will almost by definition, provide a closer approximation of the observed SED over this limited wavelength range. In the following, as for the MBBV and MBBF data fits, we will consider only the DL07 fits with $\chi_{v}^{2} \leq 2$; there are 548 of these.

\subsection{MBB fits of the DLO7 radial-profile SEDs}

We have also fit the best-fit DL07 models with MBBs. Such a procedure will not only link the physical parameters of the DL07 models (e.g., $\left.U_{\min },\langle U\rangle, q_{\mathrm{PAH}}\right)$ to the approximation of a single dust temperature, but will also explicitly demonstrate that even fixed-emissivity models such as DL07 can well approximate values of $\beta$ much lower than the intrinsic emissivity index assumed in the model.

Accordingly, for each radial SED, we have fit the best-fit DL07 models with both the variable-emissivity MBBV and the fixed-emissivity MBBF models. To each DL07 model point, we have assigned the same error as the analogous data point, and the same wavelengths have been included, namely $100 \mu \mathrm{m} \leq \lambda<$ $500 \mu \mathrm{m} . \chi_{v}^{2}$ is also calculated in the same way as for the MBBV and MBBF fits of the data themselves. Because neither model is a true observable, for these fits no color corrections have been applied to either the MBB or DL07 models. Hereafter, in order to distinguish the MBBV best-fit parameters for the data, $\beta$ and $T_{\text {dust }}$, the best-fit DL07 model MBBV values for emissivity index and dust temperature will be referred to as $\beta_{\text {DL07 }}$ and $T_{\text {dust(DL07), }}$, respectively.

Thus, as mentioned above, for each of 1166 radial SEDs in 61 galaxies, we have five fits: MBBV $+\mathrm{MBBF}$ fits of the data, DL07 fits of the data, and MBBV+MBBF fits of the best-fit DL07 models. In truth, however, we analyze the full set of five fits for 548 SEDs, because of the constraint on $\chi_{v}^{2}$ (see above).

\section{Results of the SED modeling}

An example of resulting SEDs for the annular regions of an early-type galaxy is given in Fig. 7, and the entire set of KINGFISH galaxies is shown in Appendix C. Each observed SED is plotted together with the best-fit MBBV and DL07 models ${ }^{12}$; the upper panels show the brightness panels at various wavelengths (left) and the radial run of $T_{\text {dust }}$ and emissivity in$\operatorname{dex} \beta$ (right). In the lower panels, individual data points are required to have $S / N \geq 3.0$, and the $70 \mu \mathrm{m}$ (PACS+MIPS) and $500 \mu \mathrm{m}$ points are excluded from the fits (see Sect. 5). As an example of each morphological type, consider: an elliptical, NGC 584 (Fig. 7); a lenticular NGC 1266 (Fig. C.15); a spiral NGC 6946 (Fig. C.59); and a dwarf irregular Holmberg II (Fig. C.5).

Figures 7 and C.15 clearly show that Herschel is able to trace dust well beyond $R_{\mathrm{opt}}$ even in early-type galaxies. We are able to measure dust SEDs in NGC 1266, a lenticular SB0, out to $\gtrsim 2 R_{\text {opt }}$, and in NGC 584, an E4, to $1.6 R_{\text {opt }}$. In general, the dust emission is well fit by both the MBBV and DL07 models; the DL07 models are able to accommodate fitted emissivity $\beta \lesssim 2.5$ (see, e.g., the inner regions of NGC 6946 in Fig. C.59). Moreover, the DL07 models have an average dust emissivity in$\operatorname{dex} \beta \sim 2.1$ (Bianchi 2013) but are able to reproduce low values of $\beta \sim 0.5-1$ because of the range of ISRF intensities intrinsic to the models. This behavior is particularly evident in the outer regions of NGC 6946 (Fig. C.59) and for almost the entirety of Holmberg II (Fig. C.5). Thus, low values of apparent best-fit emissivity index $\beta \lesssim 1.5$ cannot be interpreted as necessarily due to intrinsic properties of grain populations. However, the steep FIR SED slopes implied by high values $\beta \gtrsim 2.5$ are not well reproduced by the DL07 models (e.g., NGC 5457, see Fig. 10) and may be intrinsic. Possible reasons for this behavior will be discussed in Sect. 7.

\subsection{Dust optical depth and temperature}

Another way to illustrate the exponential trend of the dust in KINGFISH galaxies is with dust optical depth $\tau_{\text {dust }}$ and its dependence on galactocentric distance. Figure 8 shows $\tau_{\text {dust }}$ evaluated at $250 \mu \mathrm{m}$ (with MBBV temperatures) plotted against normalized radius, $R / R_{\text {opt }}$. Only those MBBV fits with $\chi_{v}^{2} \leq 2$ are shown, because of the need for reliable temperatures to calculate $\tau_{\text {dust }}$. The surface brightnesses in $\tau_{\text {dust }}$ have been corrected for inclination to correspond to face-on orientation. The dotted and dashed lines show a negative slope with the inverse of $R_{\mathrm{d}} / R_{\text {opt }}=0.57$, the mean over all the galaxies; this is shallower than the mean of 0.35 for only spirals discussed in Sect. 3.3. If

\footnotetext{
12 The entire suite of plots is given in Appendix C and the SED data are available at the CDS.
} 

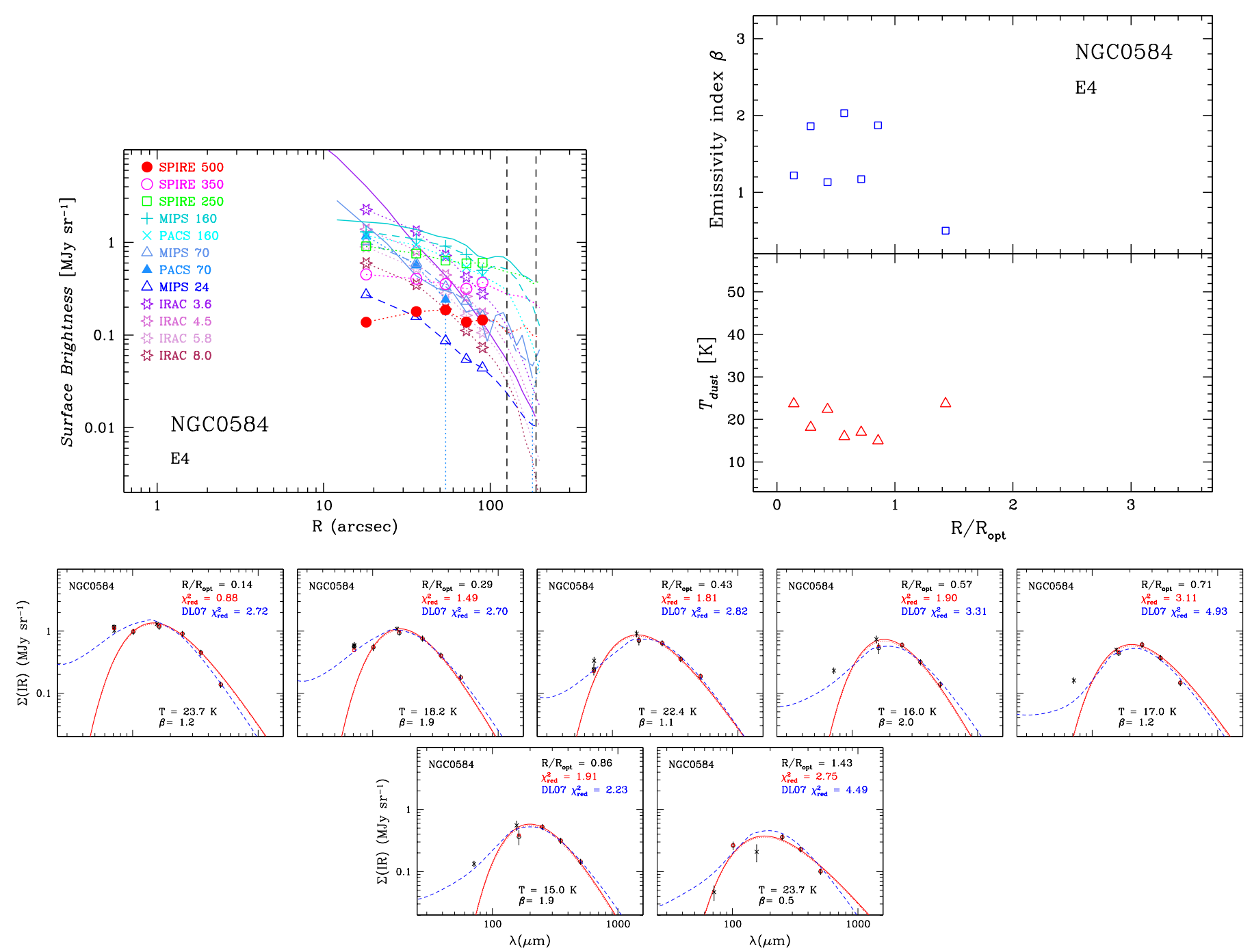

Fig. 7. NGC 584 (E4) radial profiles. Top left panel: observed surface brightness profiles, $\Sigma_{\mathrm{IR}}$, for PACS, MIPS, SPIRE, and IRAC $3.6 \mu \mathrm{m}$ shown in $\log$-log space. Symbols distinguish the different wavelengths for radial bins $\leq 100^{\prime \prime}$. The vertical dashed lines illustrate $R_{\mathrm{opt}}$ and $1.5 R_{\mathrm{opt}}$. The solid curves give the radial profiles at $3.6 \mu \mathrm{m}, 24 \mu \mathrm{m}, 70 \mu \mathrm{m}$, and $160 \mu \mathrm{m}$ from Muñoz-Mateos (priv. comm.) which were not convolved to the MIPS $160 \mu \mathrm{m}$ resolution as are the profiles presented here. Top right panel: MBBV radial temperature trends $T_{\text {dust }}$ and emissivity index trends $\beta$ vs. normalized radii, $R / R_{\text {opt }}$. Sets of fluxes to fit are defined over $\geq 4$ wavelengths. The bottom panels show each MBBV SED fit as a (red) solid line, $\Sigma_{\mathrm{IR}}$ vs. wavelength, with best-fit temperature and emissivity index $\beta$. Herschel data are shown as open circles and (red) squares with squares corresponding to the calibration corrections inferred from the MBB fits; MIPS data are shown as $\times$. As discussed in the text, neither the $70 \mu$ m nor the $500 \mu \mathrm{m}$ data points are included in the MBB fits. Also shown are the best-fit DL07 models as a (blue) dashed line. $\chi_{v}^{2}$ values are given for both fits in the upper right corner of each plot.

the surface brightnesses in the $\tau_{\text {dust }}$ calculation are not corrected for inclination, the noise in the radial trend remains roughly the same but the mean dust optical depth is raised by a factor of 2 , $\sim 0.3$ dex, as shown by the dashed regression line. Although there is much variation at small radii, the dust optical depth falls off as expected, in a roughly exponential distribution.

Like $\tau_{\text {dust }}$, dust temperature $T_{\text {dust }}$ also decreases with radius, but much more gradually. Such a trend can be appreciated in the individual upper right panels in Fig. 7, but is shown explicitly in Fig. 9 where we have plotted $T_{\text {dust }}$ vs. $R / R_{\text {opt }}$ in the left panel and against $\tau_{\text {dust }}(250)$ in the right. As in Fig. 8, only those radial SEDs with MBBV $\chi_{v}^{2} \leq 2$ are shown. The dashed regression line is derived from a linear fit of $T_{\text {dust }}$ with radius: $T_{\text {dust }}=-2.41 R / R_{\text {opt }}+21.2$. Although there is much variation, there is a significant trend for dust to be cooler in the outer regions of the KINGFISH galaxies, as also found by Galametz et al. (2012). The right panel of Fig. 9 shows that dust also tends to be cooler at low dust optical depth $\tau_{\text {dust }}$, but again with much scatter.

In some cases, very low $\tau_{\text {dust }}$ is associated with warm temperatures, perhaps associated with star-forming regions in latetype galaxies or in extended tenuous disks. We have confirmed that this effect is not merely due to sensitivity limits. The lowest $\Sigma_{250}$ surface brightnesses $(S / N \geq 3)$ measured in our sample, $\Sigma_{250} \sim 0.13-0.15 \mathrm{MJy} \mathrm{sr}^{-1}$, are associated with PACS or MIPS $160 \mu \mathrm{m}$ surface brightnesses $(S / N \geq 3) \gtrsim 0.05 \mathrm{MJy} \mathrm{sr}^{-1}$, slightly lower than what would be expected in the worst-case scenario of variable-emissivity $\mathrm{MBB}$ dust emission with $\beta=0.5$ and $T_{\text {dust }}=15 \mathrm{~K}$, namely $\Sigma_{160} \sim 0.07 \mathrm{MJy} \mathrm{sr}^{-1}$. However, the lowest values of $\Sigma_{250}$ are not necessarily those with the lowest values of $\tau_{\text {dust }}($ at $250 \mu \mathrm{m}), \lesssim 1 \times 10^{-6}$. For $\tau_{\text {dust }}$ in this range (31 radial data points), the average $\Sigma_{250}=0.5 \mathrm{MJy} \mathrm{sr}^{-1}$ (with the lowest value of $\Sigma_{250} \sim=0.13 \mathrm{MJy} \mathrm{sr}^{-1}$ as above). The mean PACS or MIPS $160 \mu \mathrm{m}$ surface brightness in this 


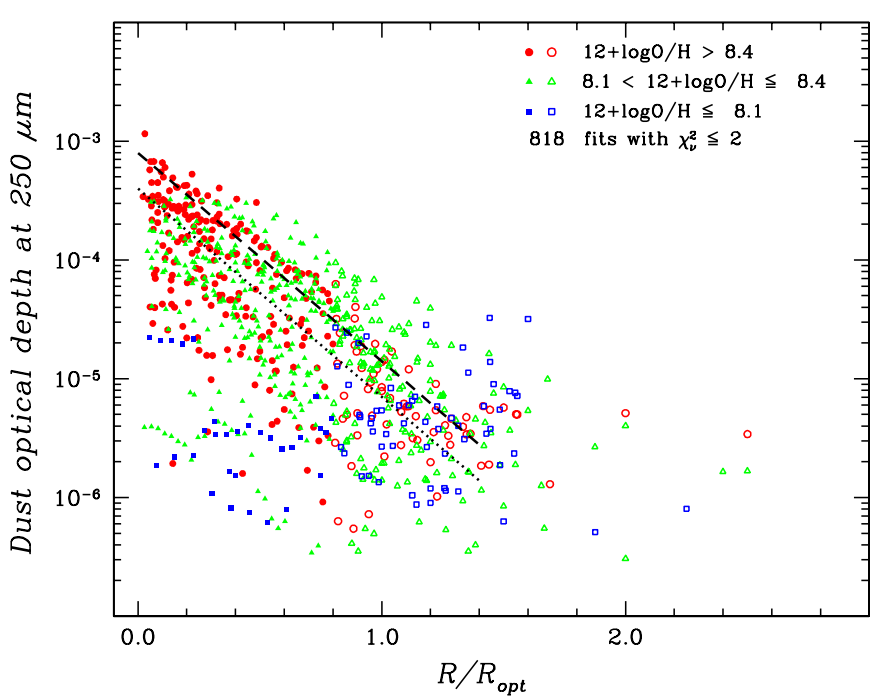

Fig. 8. Face-on $250 \mu \mathrm{m}$ optical depth $\tau_{\text {dust }}$ obtained from MBBV fits plotted against normalized radius, $R / R_{\text {opt }} ; \tau_{\text {dust }}$ has been corrected for inclination as described in the text. Points are coded by their oxygen abundance, with filled symbols corresponding to $R / R_{\text {opt }} \leq 0.8$ and open ones to larger radii. Only fits with $\chi_{v}^{2} \leq 2$ are shown. Both dashed and dotted lines show the mean $250 \mu$ m exponential scalelength $\left(R_{\mathrm{d}} / R_{\mathrm{opt}}=0.57 \pm 0.5\right)$ derived as the average over all galaxies; the offset between the two lines corresponds to the mean difference between faceon orientation (dotted line) and the observed one (dashed line, with $\tau_{\text {dust }}$ not corrected for inclination).

low $\tau_{\text {dust }}$ regime is $\sim 0.7 \mathrm{MJy} \mathrm{sr}^{-1}$, with the lowest value being $\sim 0.07 \mathrm{MJy} \mathrm{sr}^{-1}$, associated with $\Sigma_{250}=0.23 \mathrm{MJy} \mathrm{sr}^{-1}$. With dust having $\beta=0.5$ and $T_{\text {dust }}=15 \mathrm{~K}$, and this $\Sigma_{250}$, we would expect $\Sigma_{160} \sim 0.15 \mathrm{MJy} \mathrm{sr}^{-1}$, well above the lowest observed value. Thus, we conclude that the KINGFISH profile sensitivity limits are sufficient to detect, if it were present, dust with low opacity $\left(\tau_{\text {dust }} \lesssim 1 \times 10^{-6}\right)$, cold temperatures $\left(T_{\text {dust }} \sim 15 \mathrm{~K}\right)$ and very flat $(\beta=0.5)$ spectral distributions. As a result, the high-temperature, low-opacity dust shown in Fig. 9 seems real, perhaps due to the low metallicities and high values of $f_{\mathrm{PDR}}$ associated with this high- $T_{\text {dust }}$ low- $\tau_{\text {dust }}$ regime (see also Sect. 6.4 and Fig. 11).

\subsection{Radial emissivity variations}

Unlike $T_{\text {dust }}$ and $\tau_{\text {dust }}$, emissivity index $\beta$ shows no clear trend when considered globally as shown in the upper left panel of Fig. 10. The radial behavior of $\beta$ can vary significantly from one object to another. Many KINGFISH galaxies have negative radial gradients (see upper-right panel of Fig. 10). Such a trend was already observed in the late-type spiral (Scd), M 33 (Tabatabaei et al. 2014), at least over its inner disk, and is also observed in many of the late-type galaxies of our sample but not only in late-type objects: NGC 4725 and NGC 3049 are earlier spiral types (SABab, SBab, respectively). However, there are also other kinds of radial variations, for instance a $\beta$ that first rises toward $\sim R / R_{\text {opt }}$ then falls at $R \gtrsim R_{\text {opt }}$. Some of the late types (e.g., IC 342, NGC 6946, also Scd) show such "rising/falling" $\beta$ behavior (see lower-left panel of Fig. 10). Finally, some galaxies show a $\beta$ that rises almost monotonically toward large radii (see lower right panel of Fig. 10). Thus, the dependence of $\beta$ behavior on Hubble type is not clear. Nor do the trends seem to depend on metallicity, since there is no clear correlation with either emissivity index or type of radial decline. The physical meaning of $\beta$ variations will be discussed in Sect. 7 .

\subsection{Radiation field and dust heating}

Dust heating is expected to decrease with radius because of the decline of stellar surface density. The bulk of the dust in terms of mass is heated by $U_{\min }$ so we would expect an analogous radial decline of $U_{\min }$. Such a trend is shown in Fig. 11 where we have plotted $U_{\min }$ as a function of normalized galactocentric distance, as well as the other best-fit DL07 parameters, $q_{\mathrm{PAH}}, \gamma$, and $f_{\mathrm{PDR}}$. The upper left panel shows that in the KINGFISH galaxies, on average, $U_{\min }$ decreases roughly as a power law with $R / R_{\mathrm{opt}}$. We can use the relation between $U_{\min }$ and $T_{\text {dust }}$

$U_{\min }(R)=U_{0}\left(\frac{T_{\text {dust }}(R)}{T_{0}}\right)^{(4+\beta)}$

and combine it with the linear decrease of $T_{\text {dust }}$ with $R / R_{\mathrm{opt}}$ (see Sect. 6.1 and Fig. 9) to roughly predict the radial decline of $U_{\min }$. Fitting $T_{\text {dust(DL07) }}$ determined from the DL07 MBB fits to a linear trend with radius, we find $T_{\mathrm{dust}(\mathrm{DL} 07)}=-1.74 R / R_{\mathrm{opt}}+22.8$; inserting this expression into Eq. (6), fixing $\beta$ to the DL07 value of $\sim 1.8$, and setting $T_{\text {dust(DL07) }}$ to the DL07 value for $R=0$ of $\sim 22 \mathrm{~K}$ gives $U_{0}=0.93$. Equation (6) with these values is shown as the (yellow) long-dashed curve in the upper left panel of Fig. 11. The median and quartiles of the data are shown by solid and shortdashed curves respectively. The radial trend of $U_{\text {min }}$ predicted by Eq. (6) follows roughly the data, but does not capture the variation shown by the percentile curves, probably because the linear decrease of $T_{\text {dust }}$ imposed above is only a crude approximation of the radial variation of dust temperature. We have checked that the behavior at $R \gtrsim R_{\mathrm{opt}}$ is not governed by only a few galaxies; there are in fact 92 radial data points in 32 galaxies beyond this radius with DL07 $\chi_{v}^{2} \leq 2$.

\section{4. $P A H$ and $P D R$ fractions}

Figure 11 shows that there is a large spread for both the PAH fraction $q_{\mathrm{PAH}}$ and the fraction of dust exposed to ISRF $>U_{\text {min }}$ $f_{\mathrm{PDR}}$; neither parameter decreases systematically with galaxy radius. However, $q_{\mathrm{PAH}}$, shown in the upper right panel, tends to be slightly lower beyond $R_{\mathrm{opt}}$ than for $R / R_{\mathrm{opt}} \leq 1$, while $f_{\mathrm{PDR}}$, shown in the lower right, tends to be higher (the medians and quartile deviations are also shown in Fig. 11). High $f_{\mathrm{PDR}}$ could be related to the high- $T_{\text {dust }}$ low- $\tau_{\text {dust }}$ regime illustrated in Fig. 9 in which tenuous dust in the outer regions tends to be warmer, but possibly less rich in PAHs. It is possible that this radial increase in the PDR fraction is related to far-ultraviolet extended disks (e.g., Gil de Paz et al. 2005; Bigiel et al. 2010; Holwerda et al. $2012 \mathrm{~b}$ ), and the ongoing star formation associated with $\mathrm{H}$ i there (e.g., Bigiel et al. 2010; Holwerda et al. 2012b; Cortese et al. 2012).

\section{Discussion}

Much previous work has been focused on characterizing dust grain populations by fitting FIR SEDs. In particular, the MBBV approach has been used to establish that emissivity indices are not constant either globally in galaxies or in individual regions. However, as mentioned in Sect. 5.1, the interpretation of such variations is still open to debate. Our KINGFISH data allow a new perspective on how the properties of dust can be quantified in nearby galaxies. The radial profiles probe a wide range of ISRF levels; $U_{\min }$ can be as high as 20

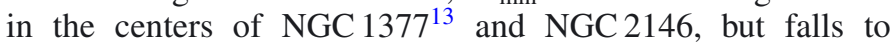

13 This galaxy is optically thick for $\lambda \lesssim 30 \mu$ m so the DL07 models may not be strictly appropriate because of the inherent assumption that the dust emission is optically thin. 


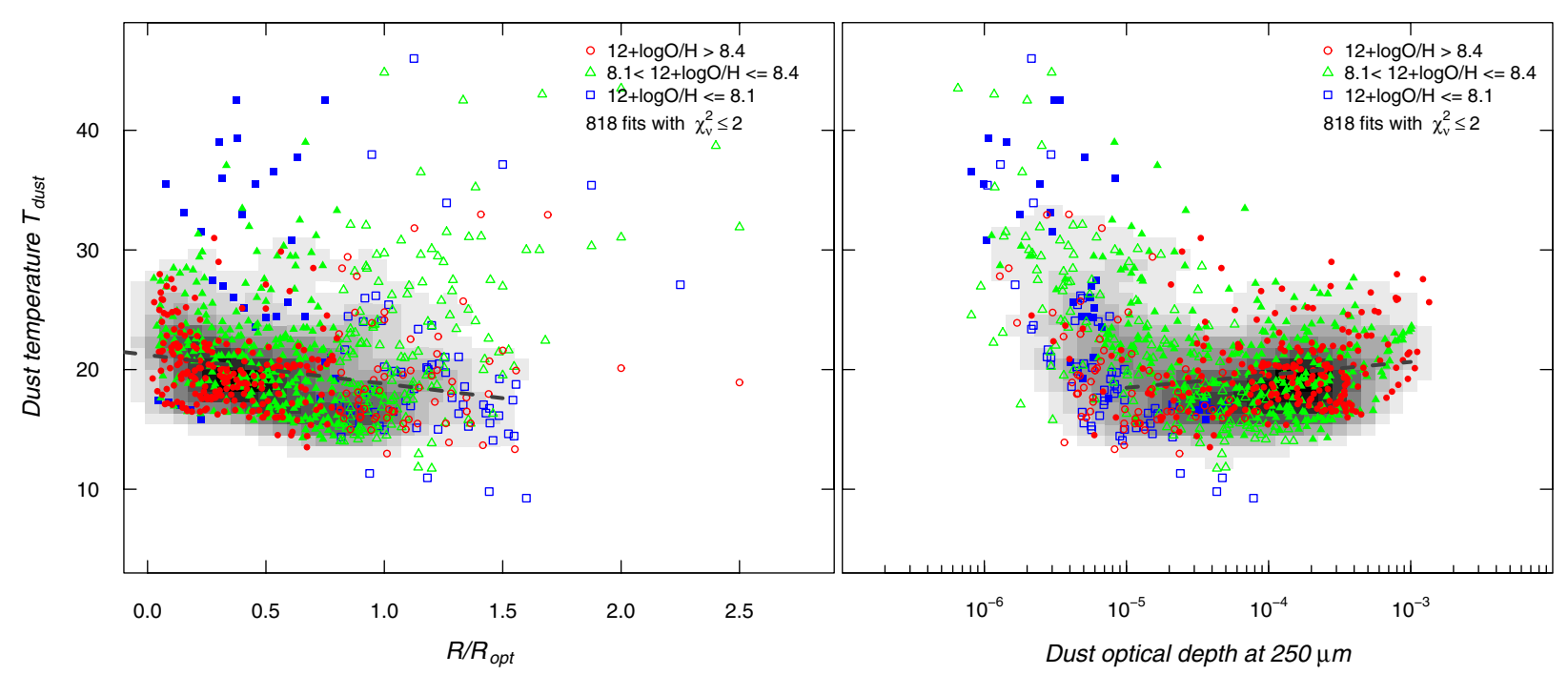

Fig. 9. Left panel: dust temperature $T_{\text {dust }}$ vs. normalized galactocentric distance $R / R_{\text {opt }} ;$ right panel: $T_{\text {dust }}$ vs. $\tau_{\text {dust }}$ evaluated at $250 \mu$ m. As in Fig. 8 , points are coded by their oxygen abundance, with filled symbols corresponding to $R / R_{\mathrm{opt}} \leq 0.8$ and open ones to larger radii. The underlying grayscale shows the two-dimensional density distribution of the data. The dashed lines are unweighted linear regressions to the data points, excluding outliers with $T_{\text {dust }} \geq 30 \mathrm{~K}$ (in the left panel, $T_{\text {dust }}=-2.41 R / R_{\text {opt }}+21.2$ ).

$U_{\min }=0.1$ in the centers of dwarf galaxies (e.g., DDO 154, DDO 165, Holmberg II, IC 2574, M 81 Dwarf B) and in the outskirts of virtually all spirals and early-type galaxies. Thus, our sample can quantify trends with $\beta$ and $T_{\text {dust }}$ over a factor of $\sim 200$ in ISRF intensities: from 10 times lower than the solar neighborhood value to almost 20 times higher. This section is devoted to an analysis of the relation of fitted emissivity indices $\beta$, temperature $T_{\text {dust }}$, and the parameters of the DL07 models, together with the insights they give about the physical characteristics of dust emission.

\subsection{The temperature-emissivity degeneracy}

MBBV fits of FIR dust SEDs are notorious for the degeneracy between dust temperature $T_{\text {dust }}$ and apparent emissivity index $\beta$ (e.g., Galametz et al. 2012, see also Sect. 5.1). As mentioned before, such trends have been generally interpreted as spurious, a signature of temperature mixing along the LOS combined with uncertainties in flux measurements, rather than intrinsic variations in dust properties (e.g., Shetty et al. 2009a,b; Juvela \& Ysard 2012; Juvela et al. 2013). Indeed, Kirkpatrick et al. (2014) showed that by limiting analysis to data with high $S / N(\geq 10)$ it is possible to significantly reduce the correlation between $T_{\text {dust }}$ and $\beta$.

The relation between these two parameters in our MBBV fits to the KINGFISH profiles and in the MBBV fits of the analogous best-fit DL07 models is shown in Fig. 12. Despite our grid technique to mitigate the degeneracy between $\beta$ and $T_{\text {dust }}$, there is still some vestige of it remaining in our MBBV fits. The left panel of Fig. 12 shows that higher values of $\beta$ tend to be associated with lower $T_{\text {dust }}$. However, for $\beta=0.5$, the lowest value allowed by our fitting algorithm, $T_{\text {dust }}$ can be as low as $\sim 14 \mathrm{~K}$ and as high as $z 40 \mathrm{~K}$. Thus, our fits partly reduce the known degeneracy between these parameters because they do not rely on traditional curve fitting. In Fig. 12, data points are coded by metallicity and the most metal-rich inner regions (filled circles) of KINGFISH galaxies generally have $\beta \gtrsim 2$.

The right panel of Fig. 12 shows the MBBV fits to the bestfit DL07 models. The MBBV DL07 $\beta_{\text {DL07 }}$ never exceed $\beta \sim 2$, as expected because of the intrinsic nature of the DL07 grain populations. Because the "canonical" $\beta-T_{\text {dust }}$ degeneracy is virtually absent in the MBBV DL07 fits, we conclude, in accordance with previous work, that data uncertainties play an important role its generation. The MBBV fits in the left panel of Fig. 12 show, if anything, an increase in $\beta$ with decreasing $T_{\text {dust }}$; the MBBV fits to the DL07 models in the right panel show, if anything, an increase of $\beta$ with increasing $T_{\text {dust }}$. The DL07 models, by definition, reproduce a range of dust temperatures through the distribution of ISRF intensities, so if this were the primary cause of the degeneracy in the data, we would expect to see the same trends in the $\beta-T_{\text {dust }}$ relation for the data and the best-fit DL07 models and we do not. However, the MBBV fits of the DL07 models are restricted to a narrower range of temperatures than the MBBV fits to the data; $\sim 56 \%$ of the MBBV fits to the data with $\chi_{v}^{2} \leq 2$ have $T_{\text {dust }} \leq 20 \mathrm{~K}$, but only $\sim 25 \%$ of the MBBV fits to the DL07 models have similarly low temperatures. Nevertheless, the data suffer from measurement noise and perhaps combinations of $T_{\text {dust }}$ and $\beta$ that are not present in the DL07 models. Hence, we cannot draw definitive conclusions from these trends.

Figure 13 compares the emissivities ( $\Delta \beta$ relative to $\beta=2$ ) from the MBBV fits with temperature $T_{\text {dust }}$. The left panels show $T_{\text {dust }}$ derived from MBBF fits, and the right panels the difference $\Delta T_{\text {dust }}$ of the temperature from variable- $\beta$ fits and fixed $\beta$ fits. The $\beta_{\text {DL07 }}$ of the DL07 best-fit models never exceeds $\sim 2$, while the data sometimes need $\beta \sim 3$; hence the upper panels with the data show a larger excursion in $\Delta \beta$ than the lower ones (with the DL07 best-fit models). The upper left panel of Fig. 13 gives a trend of $\Delta \beta$ with $T_{\text {dust }}$ derived from fixed- $\beta$ MBBF fits that is similar to the trends of the DL07 models in the lower panels. The upper right panel show that MBBF temperatures exceed those in MBBV fits for $\beta \geq 2$, while they fall below them for lower values of $\beta$.

Such behavior of warmer $T_{\text {dust }}$ from MBBF fits with increasing $\Delta \beta$ would be expected if temperature mixing were at work in the data. First, single-temperature MBBF fits are as reasonable a representation of the observed SEDs as the MBBV ones; $\sim 89-90 \%$ of the observed SEDs with good S/N are well approximated by both kinds of fits ( $827 / 920$ vs. $818 / 920$, see Sect. 5.2). This is perhaps a surprising result given the additional 


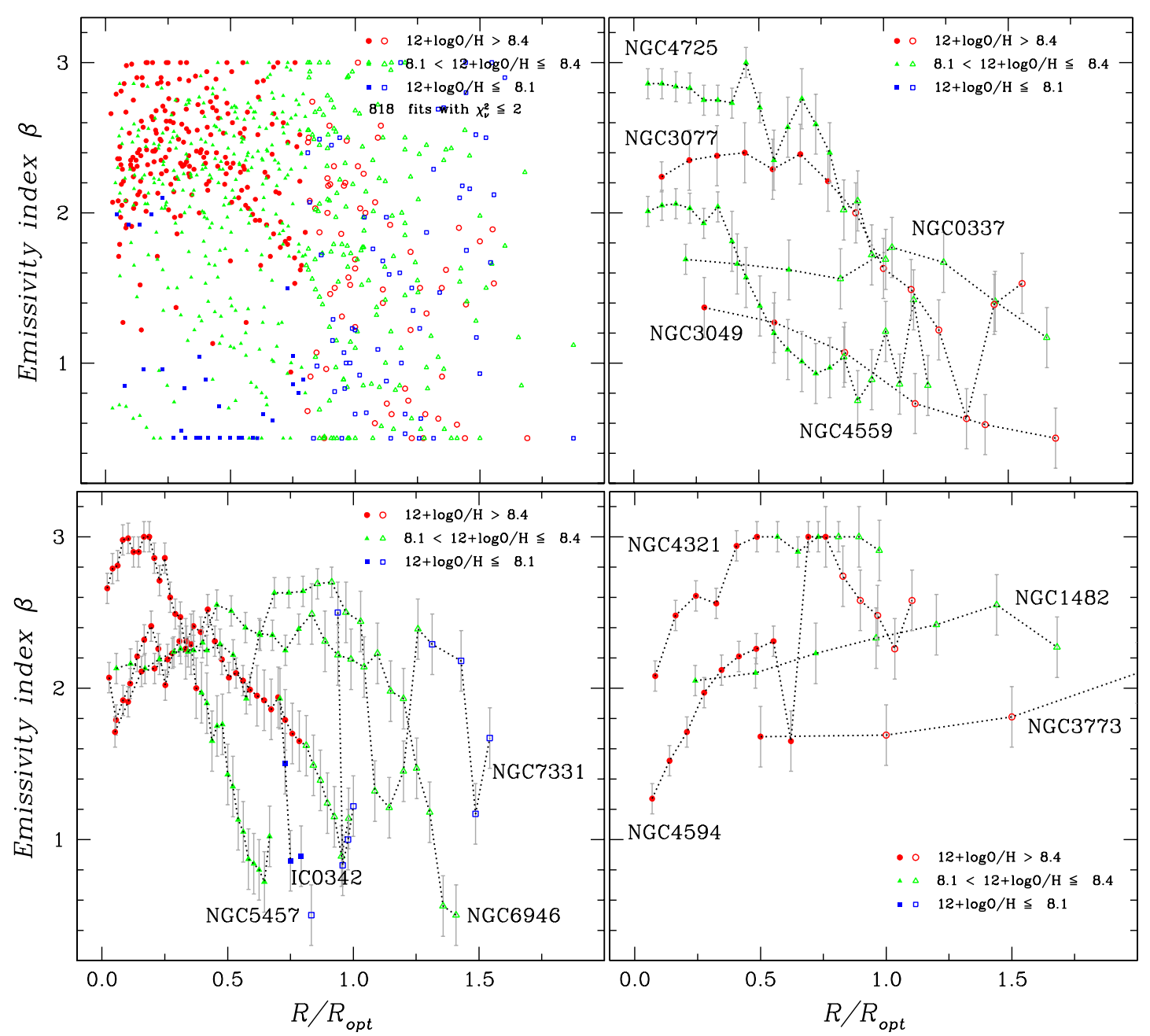

Fig. 10. Emissivity index $\beta$ gradients with galactocentric distance $R / R_{\text {opt }}$. The upper-left panel shows all galaxies together, while individual galaxies are shown in the remaining panels. The upper-right panel illustrates examples of negative radial gradients, the lower-left rising then falling, and the lower-right panel positive radial gradients. In all panels, as in Fig. 8, points are coded by their oxygen abundance, with filled symbols corresponding to $R / R_{\mathrm{opt}} \leq 0.8$ and open ones to larger radii.

free parameter in the MBBV fits. Second, single-temperature MBBV fits tend to compensate for broader SEDs by flattening $\beta$, which because of noise and the mathematical form of the MBB function causes warmer temperatures (that peak at shorter wavelengths) to be associated with flatter $\beta$, thus creating the "canonical" degeneracy of $T_{\text {dust }}$ and $\beta$. However, if the data were truly encompassing a range of temperatures at long wavelengths, fixed- $\beta$ MBBF fitting would result in a trend similar to that observed in the upper right panel of Fig. 13. This is because our wavelength coverage combined with an apparently shallower (than $\beta=2$, below dotted line) observed slope makes the MBBF fit try to compensate by lowering $T_{\text {dust }}$ (moving the peak towards longer wavelengths); this would make the curve around the peak emission broader than it would be at higher temperatures farther from the peak toward the blue. On the other hand, the MBBF fit would compensate an apparently steeper slope by pushing the peak toward shorter wavelengths, thus raising the fitted $T_{\text {dust }}$. We investigate this point further in the next section.

\subsection{Temperature mixing along the line of sight}

We first want to establish whether observed trends of emissivity index $\beta$ can be attributed to temperature variations along the
LOS. Following Paradis et al. (2012), we have calculated the average variation with wavelength of dust emissivities in terms of the optical depth $\tau_{\text {dust }}$. We calculate $\tau_{\text {dust }}$ as the surface brightness $I_{v}\left(=F_{v} / \Omega\right)$ at each wavelength divided by the blackbody at the best-fit fixed- $\beta$ MBBF $T_{\text {dust }}$. Like Paradis et al. (2012), for the derivation of $\tau_{\text {dust }}$ we have derived the temperature $T_{\text {dust }}$ using MBBF fits with $\beta \equiv 2$ (see Sect. 5.1). If temperature mixing is in truth causing the trends between $\beta$ and $T_{\text {dust }}$, we would expect the DL07 models to show the same behavior as the data because in these models there is a multitude of cool-dust temperatures for every LOS. Accordingly, we have calculated the spectral trend of $\tau_{\text {dust }}$ also for the best-fit DL07 models, again using MBBF fits with $\beta_{\text {DL07 }} \equiv 2$; in addition to the usual $\chi_{v}^{2}$ requirement, we also specify that both PACS fluxes $(100 \mu \mathrm{m}$ and $160 \mu \mathrm{m}$ ) have $S / N \geq 3$ (see Sect. 5.2). The results are shown in Fig. 14; the left panel shows the data and the right panel the best-fit DL07 models. For the figure, only the radial points with $\chi_{v}^{2} \leq 2$ have been binned in temperature, and normalized to $\tau_{\text {dust }}$ at $160 \mu \mathrm{m}$; there are fewer fits with $\chi_{v}^{2} \leq 2$ (693 vs. 818) because of the additional requirement of PACS points with $S / N \geq 3$.

Figure 14 shows an interesting feature in both the data and the DL07 models; the slope of the FIR spectrum is flatter toward longer wavelengths similarly to the Galaxy data analyzed 

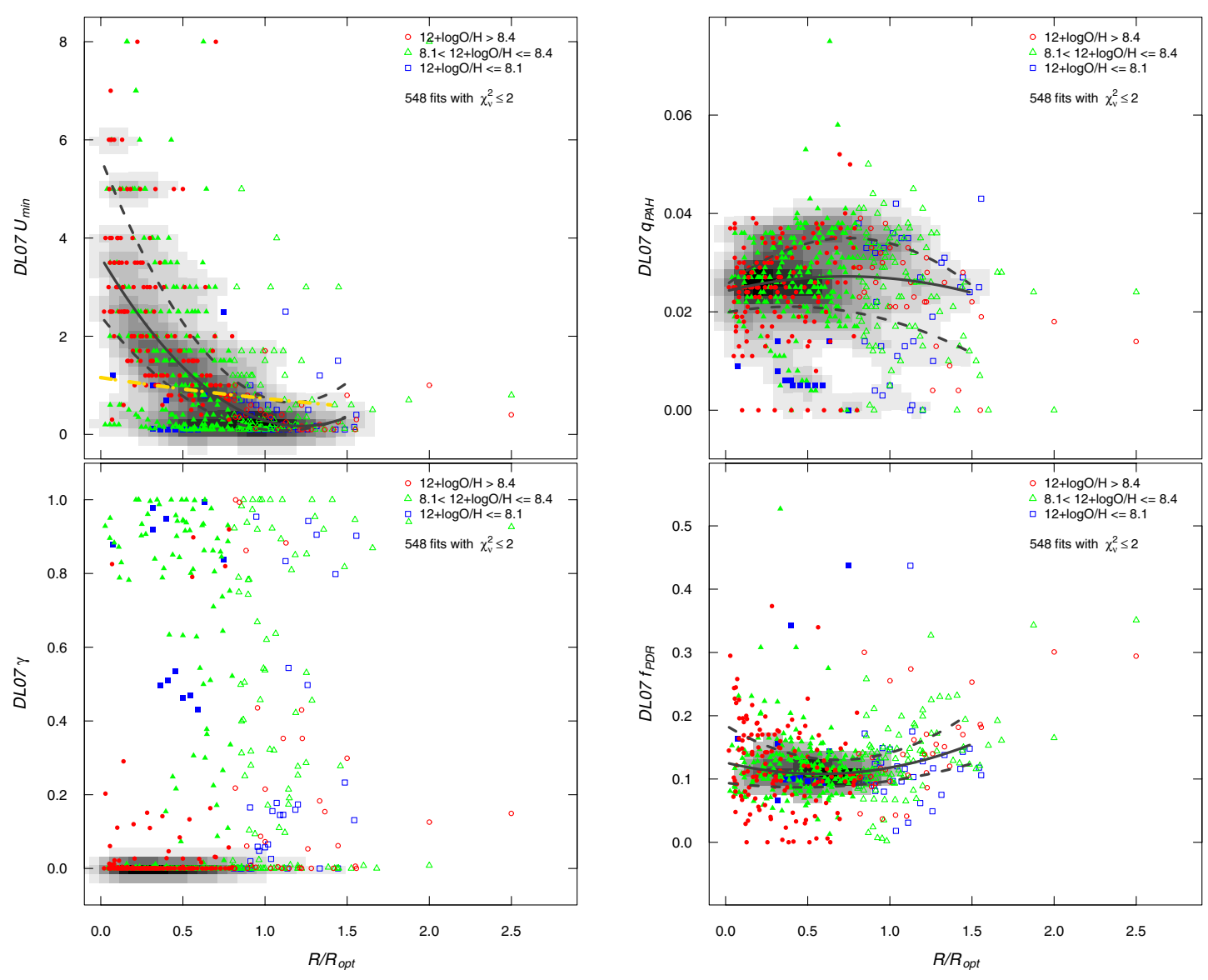

Fig. 11. Best-fit DL07 parameters plotted against normalized galactocentric distance. The upper left panel shows $U_{\min }$; the upper right $q_{\mathrm{PAH}}$; the lower left $\gamma$; and the lower right $\mathrm{PDR}$ fraction $f_{\mathrm{PDR}}$. The underlying grayscales in each panel show the two-dimensional density distributions of the data. The upper left and right panels show the mean (solid curve) and quartiles (dashed curves) of $U_{\min }$ (upper left), $q_{\mathrm{PAH}}\left(\right.$ upper right) and $f_{\mathrm{PDR}}$ (lower right). The (yellow) long-dashed curve in the upper left panel shows the relation for $U_{\min }=U_{0}\left(T_{\mathrm{dus}} / T_{0}\right)^{(4+\beta)}$ for $U_{0}=0.93, T_{0}=22 \mathrm{~K}$, and $\beta=1.8$ as described in the text. As in previous figures, (red) circles correspond to $12+\log (\mathrm{O} / \mathrm{H})>8.4$, (green) triangles to $8.1<12+\log (\mathrm{O} / \mathrm{H}) \leq 8.4$, and (blue) squares to $12+\log (\mathrm{O} / \mathrm{H}) \leq 8.1$; filled symbols indicate $R / R_{\text {opt }}<0.8$.
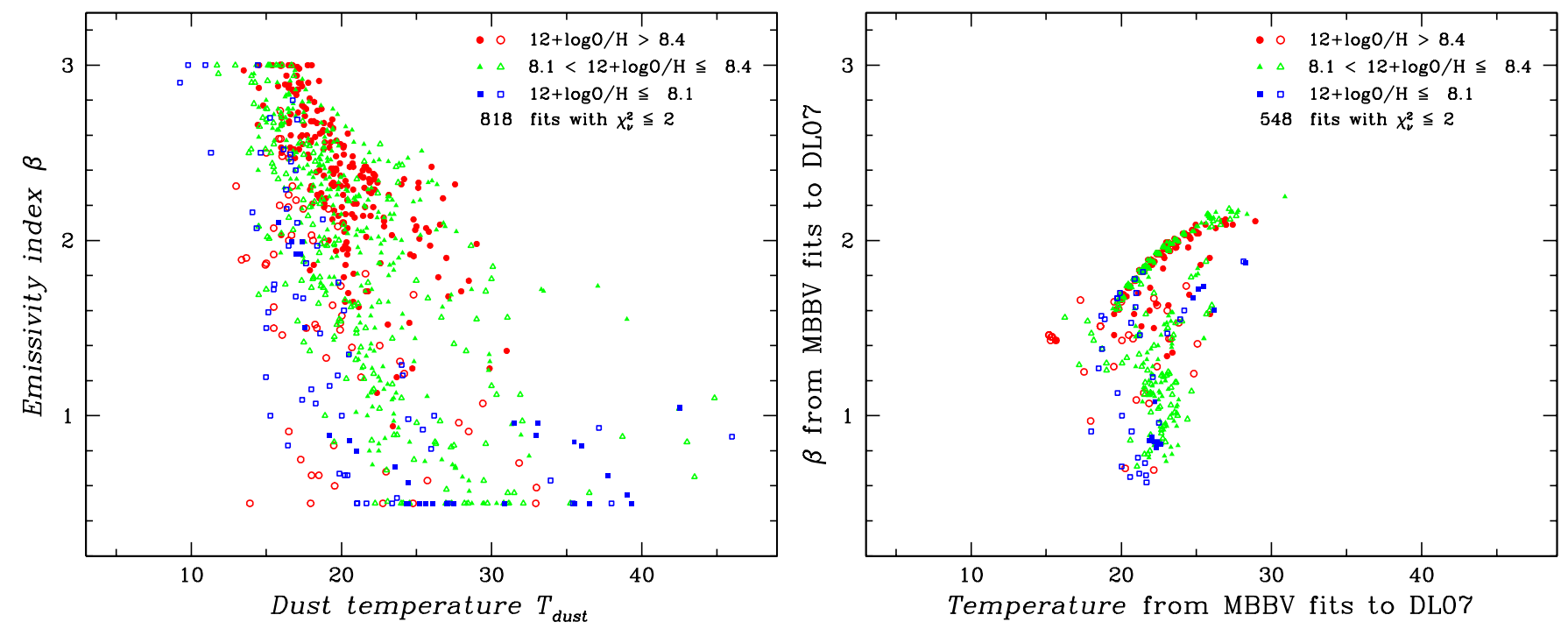

Fig. 12. Fitted MBBV emissivity index $\beta$ plotted against fitted dust temperature $T_{\text {dust }}$. The left panel shows the MBBV fits to the data, and the right the MBBV fits to the best-fit DL07 models. Data are distinguished by $\mathrm{O} / \mathrm{H}$ with (red) circles showing $12+\log (\mathrm{O} / \mathrm{H})>8.4$, (green) triangles $8.0<12+\log (\mathrm{O} / \mathrm{H}) \leq 8.4$, and (blue) squares $12+\log (\mathrm{O} / \mathrm{H}) \leq 8.1$. Filled symbols correspond to positions with normalized (to optical radius $R_{\text {opt }}$ ) radii within $R / R_{\mathrm{opt}} \leq 0.8$, and open symbols to larger radii. 

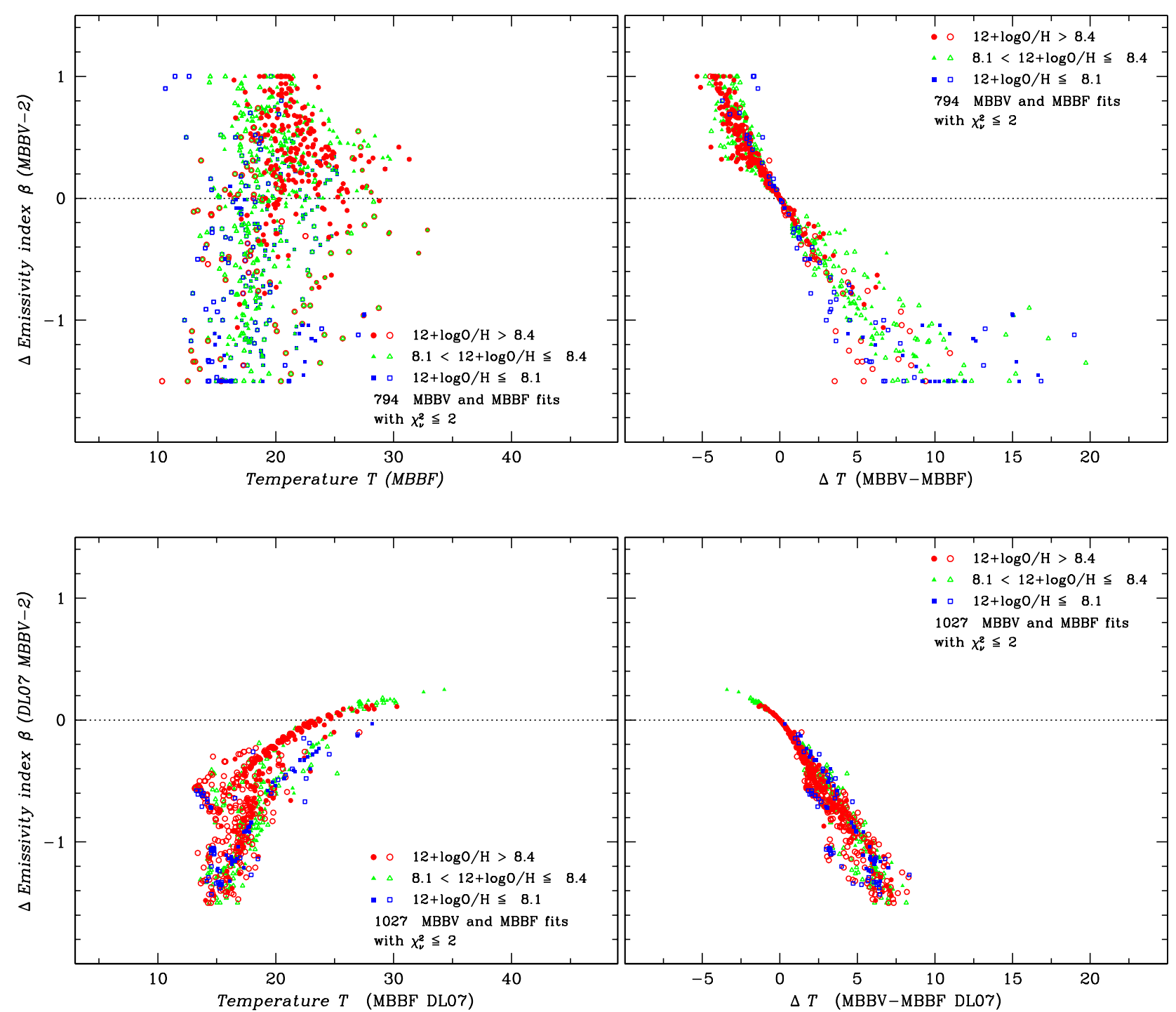

Fig. 13. Top left panel: difference of $\beta$ from the MBBV fits to the data and MBBF with $\beta=2$ vs. temperature from MBBF fits. Top right panel: the same but vs. the difference in MBBV $T_{\text {dust }}-\mathrm{MBBF} T_{\text {dust }}$. Bottom panels: the same as for the top panels but for the MBBV and MBBF fits to the DL07 best-fit models. Points are coded by their oxygen abundance, with filled symbols corresponding to $R / R_{\text {opt }} \leq 0.8$ and open ones to larger radii. Only those MBBV and MBBF fits with $\chi_{v}^{2} \leq 2$ are shown.

by Paradis et al. (2012). However, instead of the flatter slope occurring at high temperatures $\left(T_{\text {dust }} \gtrsim 30 \mathrm{~K}\right)$, in the KINGFISH data flatter $\beta$ is associated with low temperatures ( $\left.T_{\text {dust }} \lessgtr 20 \mathrm{~K}\right)$. This is the opposite of the trend expected from the $\beta-T_{\text {dust }}$ degeneracy curve which has lower $\beta$ associated with high $T_{\text {dust }}$ (see Fig. 12), but similar to the trend of flatter $\beta$ and lower $T_{\text {dust }}$ seen in the upper left panel of Fig. 13. The difference between Figs. 12 and 14 is that the parameters in the former come from MBBV fits and in the latter from MBBF fits with $\beta=2$ as illustrated in Fig. 13.

We find that the best-fit DL07 models show the same behavior as the data, namely flatter slopes at longer wavelengths corresponding to low $T_{\text {dust }}$ (see Figs. 12-14). This would imply that temperature mixing is causing the trend in the data because of the similarity of the behavior of $T_{\text {dust }}$ and $\beta$. Thus our results contrast with the conclusions of Paradis et al. (2012) who found that the Dale \& Helou (2002) models did not show the same behavior as their data for Galaxy and thus that temperature mixing along the LOS was not at work. We propose that the KINGFISH profiles probe a wider range of physical conditions than was possible with previous data. In addition, the differences may be caused by the different assumptions for $\mathrm{d} M / \mathrm{d} U$ (see Eq. (5) for the DL07 approach) of the Dale \& Helou (2002) models which are missing the bulk heating with $U_{\min }$.

Nevertheless, the spatial scales over which dust properties are integrated must play a role in the degree of temperature mixing; the observations of Paradis et al. (2012) are along the Galactic plane and KINGFISH data are averaged over $\sim 1.9 \mathrm{kpc}$ at the median sample distance (see Sect. 3). Temperature mixing is expected to be less important when the lines of sight are averaged over spatial scales commensurate with less complex dust heating. In the next section we compare $U_{\min }, T_{\text {dust }}$, and $\beta$ to try to better understand these trends between $\beta$ and $T_{\text {dust }}$.

\subsection{Radiation field, apparent emissivity index, and dust temperature}

We have seen in Sect. 6 that the radial trends of DL07 $U_{\text {min }}$ and $T_{\text {dust(DL07) }}$ are related in a power-law fashion, as expected 


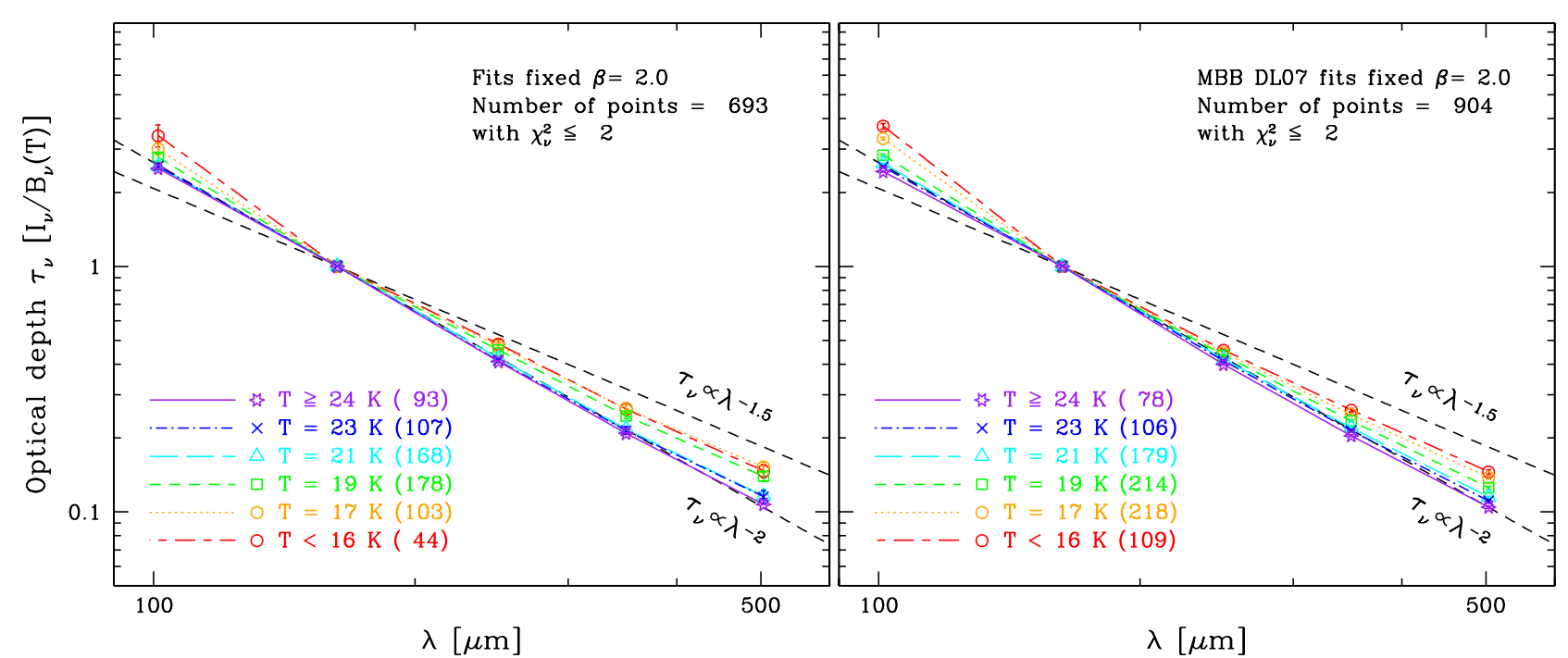

Fig. 14. Optical depth $\tau_{\text {dust }}$ normalized by $\tau_{160 \mu \mathrm{m}}$ as a function of wavelength $\lambda$. The left panel shows the data, and the right panel the DL07 best-fit models. Temperatures for the $\tau_{\text {dust }}$ calculation were obtained from MBBF fits with $\beta=2$, and are shown binned to $2 \mathrm{~K}$, with the numbers of radial points are given in parentheses. Only those MBBF fits with $\chi_{v}^{2} \leq 2$ are considered in the calculations. The dashed lines correspond to two values of $\beta=1.5,2$.

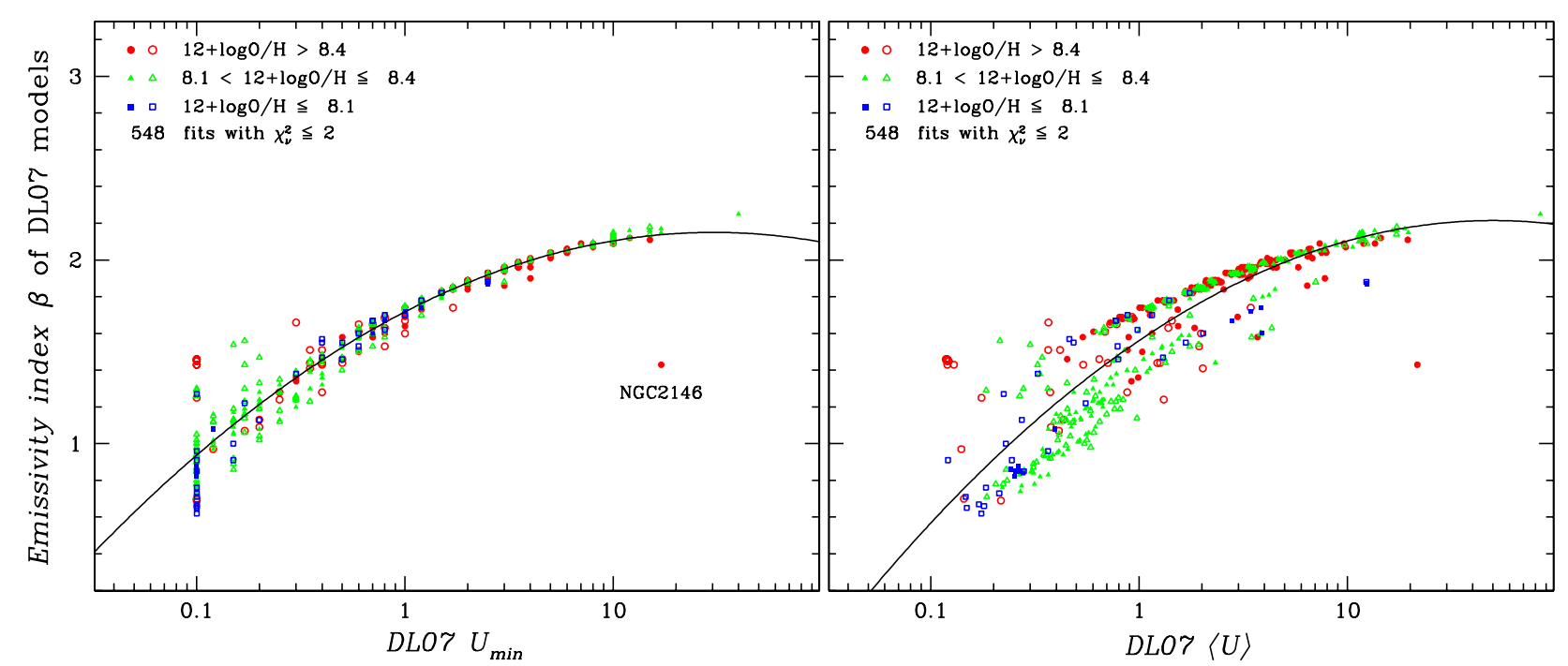

Fig. 15. Best-fit DL07 emissivity index $\beta_{\mathrm{DL} 07}$ plotted against $U_{\min }$ (in the left panel) and $\langle U\rangle$ (in the right). Only those MBBV DL07 fits with $\chi_{v}^{2} \leq 2$ are considered in the plot. As before, points are coded by oxygen abundance. The curve in the left panel is the second-order polynomial best-fit of DL07 MBBV $\beta$ to $\log _{10}\left(U_{\min }\right): \beta_{\text {DL07 }}=1.72+0.58 \log \left(U_{\min }\right)-0.20 \log ^{2}\left(U_{\min }\right)$. The right panel shows the analogous curve for $\log _{10}(\langle U\rangle)$ : $\beta_{\text {DL07 }}=1.56+0.77 \log (\langle U\rangle)-0.23 \log ^{2}(\langle U\rangle)$.

for dust in thermal equilibrium with the ambient radiation field, $T_{\text {dust }} \propto U_{\min }^{[1 /(4+\beta)]}$ (see curves in Fig. 11). Here we explore whether the emissivity index of the best-fit DL07 models $\beta_{\text {DL07 }}$ is related to $U_{\min }$ in an analogous way. Such a connection would be expected given the relation between $T_{\text {dust(DL07) }}$ and $U_{\min }$, and would help explain how the DL07 models can produce an apparent emissivity index $\beta \lesssim 1$. Figure 15 plots $\beta_{\text {DL07 }}$ against $U_{\min }$. The two parameters are closely related, as shown by the best fit second-order polynomial in the left panel of Fig. 15: $\beta_{\mathrm{DL} 07}=1.72+0.58 \log \left(U_{\min }\right)-0.20 \log ^{2}\left(U_{\min }\right)$. The right panel of Fig. 15 shows the variation of $\beta_{\mathrm{DL} 07}$ with $\langle U\rangle$; the best-fit curve is $\beta_{\mathrm{DL} 07}=1.56+0.77 \log (\langle U\rangle)-0.23 \log ^{2}(\langle U\rangle)$. The mean of the residuals for $\beta_{\mathrm{DL} 07}$ from the $U_{\min }$ curve is 0.1 ; thus for dust that behaves like the dust in KINGFISH galaxies, it is possible to estimate $\beta$ from $U_{\min }$, and vice versa. The single outlier, the central point of NGC 2146, does not follow this trend, but this galaxy contains a dusty outflow along the minor axis (Heckman et al. 2000; Kreckel et al. 2014) so these (optically thin) models may not be applicable.

Despite the mean emissivity index $\beta \sim 2$ of the DL07 models, through low values of $U_{\min }$ they are very good at imitating flat FIR-submm SEDs with apparent $\beta \lesssim 1$. Most of the variation in $\beta_{\mathrm{DL} 07}$ is for $U_{\min } \lesssim 1$, a regime which was not well sampled by previous data; the wide range in $U_{\text {min }}$ covered by the KINGFISH profiles lets this result emerge. At low $U_{\min }$, there is a larger fraction of cool dust that emits at longer wavelengths; this tends to shift the peak wavelength, broaden the SED, and flatten its apparent FIR-submm slope to $\beta \lesssim 1$. At high $U_{\text {min }}$, most of the dust is warmer, emitting radiation toward shorter wavelengths ( $\$ 200 \mu \mathrm{m}$ ) thus causing the slope of the FIR-submm SED to assume its "native" value of $\beta \sim 2$. Both the shifting of the peak wavelength and the broadening of the SED contribute to the apparently flatter slopes; when the SED peaks at longer wavelengths, we are no longer in the Rayleigh-Jeans regime where 

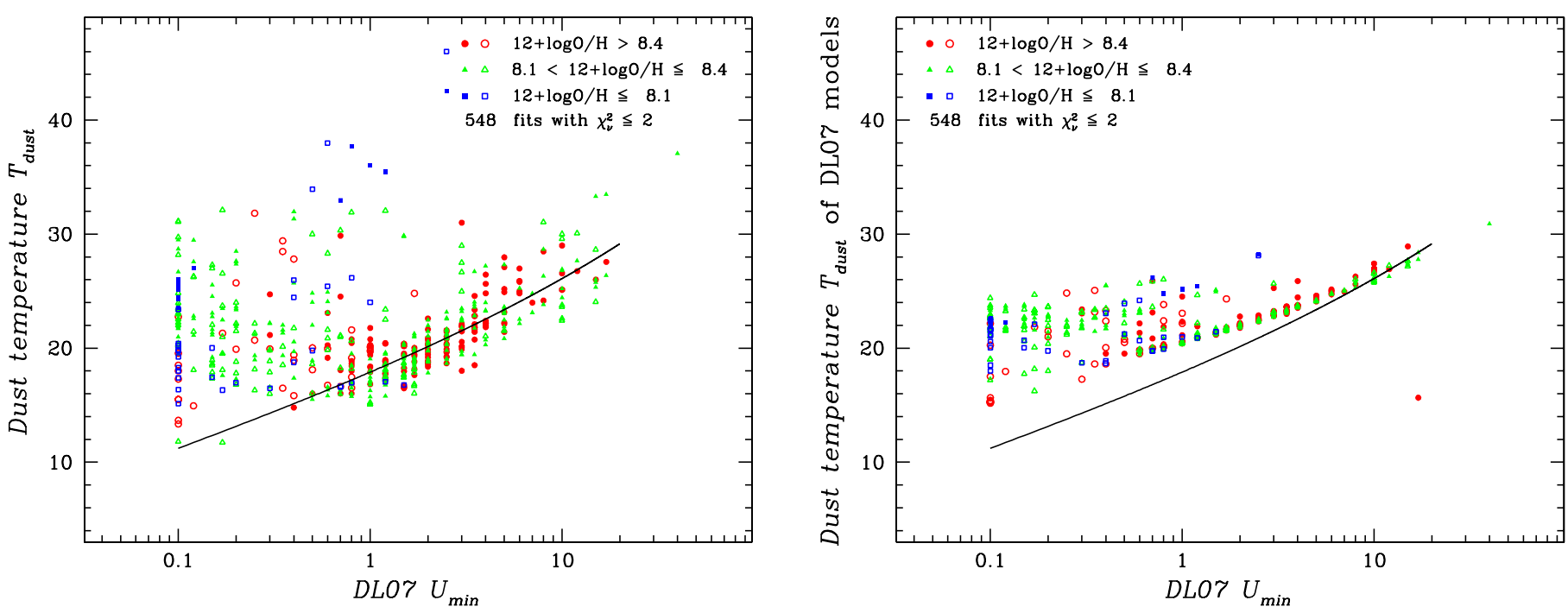

Fig. 16. Best-fit MBBV data dust temperature $T_{\text {dust }}$ (in the left panel) and best-fit MBBV DL07 $T_{\text {dust }}$ (in the right) plotted against $U_{\text {min }}$. Only those MBBV DL07 fits with $\chi_{v}^{2} \leq 2$ are considered in the plot. As before, points are coded by oxygen abundance. The curves in both panels are $T_{\text {dust }} \propto U_{\min }^{[1 /(4+\beta)]}$ assuming the relation between $\beta_{\mathrm{DL} 07}$ and $U_{\min }$ (see text and Fig. 15).

slopes should reflect true grain emissivities. The association between $\beta$ and $\langle U\rangle$ is looser than for $U_{\min }$, presumably because most of the ISM emitting dust in galaxies tends to be heated by an ISRF around $U_{\min }$ rather than $\langle U\rangle$.

Because low $U_{\min }$ would be expected to correspond to low $T_{\text {dust }}\left[\right.$ or $T_{\text {dust(DL07) }}$, we can understand the trend of flatter SEDs with cooler temperatures shown in Fig. 14. This is also illustrated in Fig. 16 where dust temperature is plotted against $U_{\min }$ : $T_{\text {dust }}$ from MBBV fits to the data are given in the left panel, and $T_{\text {dust(DL07) }}$ from MBBV fits to the DL07 models in the right. The curves in Fig. 16 correspond to $T_{\text {dust }} \propto U_{\min }^{[1 /(4+\beta)]}$, assuming the polynomial curve relating $\beta_{\mathrm{DL} 07}$ and $U_{\min }$ shown in the left panel of Fig. 15. At low values of $U_{\min }$, there is a large range of $T_{\text {dust }}$ as shown particularly in the left panel where $T_{\text {dust }}$ from data fits is plotted. However, for $U_{\min } \gtrsim 1$, the bulk of the data is close to the curve; $T_{\text {dust }}$ is expected to be lower for lower $U_{\min }$ (and equivalently for lower $\beta$ as shown in Fig. 15). The DL07 dust temperatures $T_{\text {dust(DL07) }}$ (right panel of Fig. 16) all exceed the curve (except for large values of $U_{\min } \sim 10$ ), and the trend of low $U_{\text {min }}$ and low $T_{\text {dust(DL07) }}$ (and $\beta_{\text {DL07 }}$ ) is less pronounced. Such behavior is consistent with the narrower range of the DL07 MBBV temperatures relative to the data shown in Fig. 13 and discussed in Sect. 7.1.

\subsection{Potential causes of temperature mixing}

Like much previous work (e.g., Galametz et al. 2012; Tabatabaei et al. 2014; Kirkpatrick et al. 2014), we have found radial variations of $\beta$ and $T_{\text {dust }}$. In the KINGFISH profiles, we also find radial variations of $U_{\min }$, consistent with what would be expected from considering the effects of dust reprocessing on $\beta$ and $T_{\text {dust }}$; taking all galaxies together, $\beta$ and $T_{\text {dust }}$ vary with a power-law dependence on $U_{\min }$. We have shown that in the KINGFISH profiles taken individually, $\beta$ and $T_{\text {dust }}$ are weakly related in the usual degeneracy with low values of $\beta$ (flatter slopes) associated with high values of $T_{\text {dust }}$. Nevertheless, when the MBBF fits to the profiles are binned in temperature, flatter $\beta$ corresponds to lower $T_{\text {dust }}$ (see Fig. 14). Moreover, despite their average dust emissivity index $\beta \sim 2$ the DL07 models are able to reproduce quite well the SEDs with apparently flatter slopes $\beta \lesssim 1$; this is

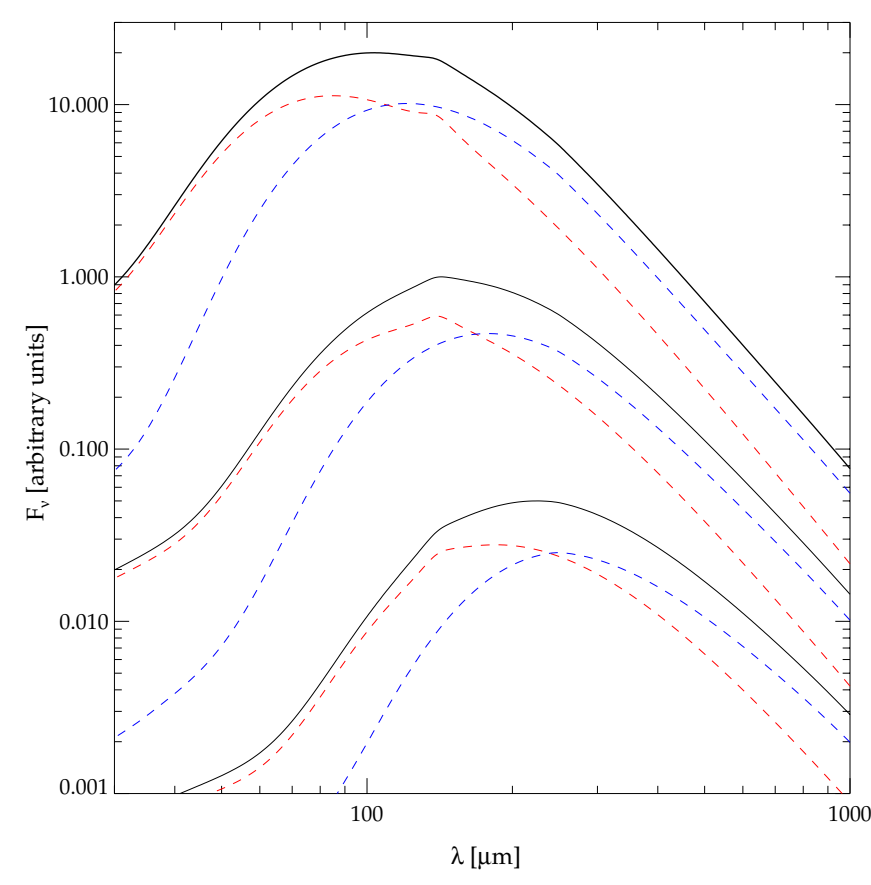

Fig. 17. SEDs of DL07 models with ISRF $\equiv U_{\min }$. Three values of $U_{\min }$ are shown: from lower to upper curves $U_{\min }=0.1,1.0,10.0$. Total emission is given by the solid (black) curve. The blue dashed curves correspond to the contribution from silicates and the red to carbonaceous grains (including graphite, ionized and neutral PAHs).

because decreasing ISRF intensities with $U_{\min } \lesssim 1$ produce dust SEDs with increasingly flat apparent emissivity indices achieving $\beta \lesssim 1$ for $U_{\min } \approx 0.1$.

Clearly apparently flat $\beta$ SEDs can be achieved with low $U_{\min }$ even with the DL07 dust models that have much higher intrinsic emissivities. We have argued that the reason for this is temperature mixing, and here we examine two separate phenomena which could be driving the mixing: the ISRF and grain composition. First, a spread of temperatures is caused by the distribution of the intensities of the ISRF heating the dust. Low $U_{\text {min }}$ means that a larger fraction of the dust tends to be cooler, with SEDs that peak at longer wavelengths $(\lambda \gtrsim 200 \mu \mathrm{m}$, see also 


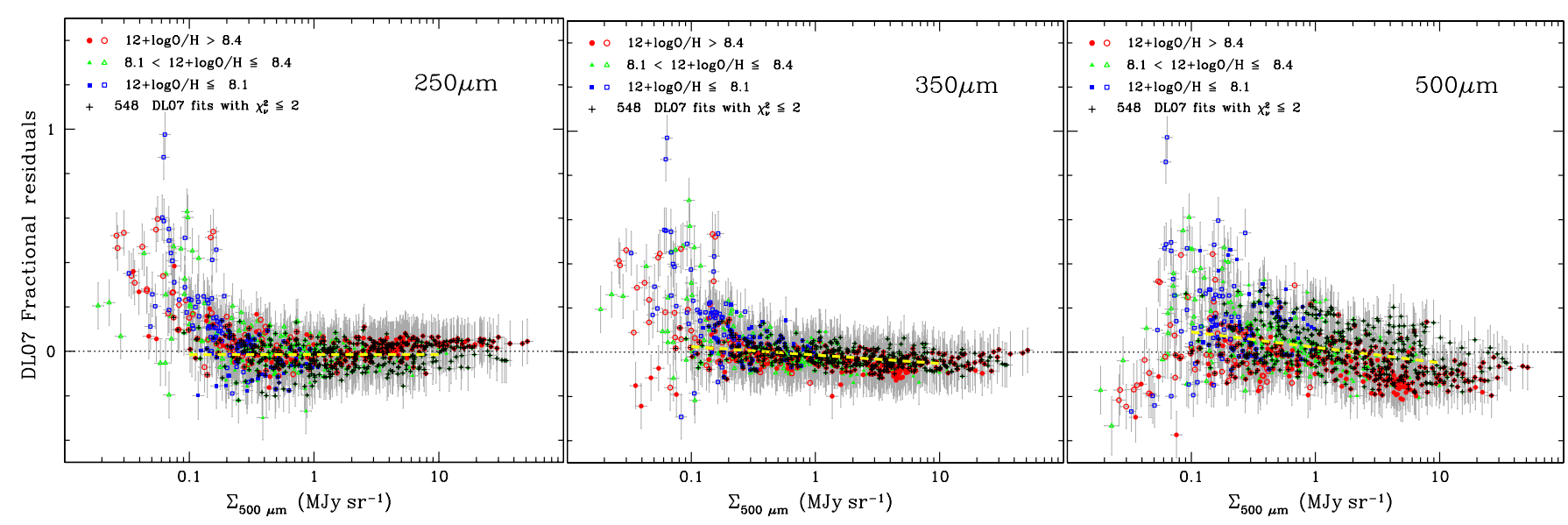

Fig. 18. SPIRE fractional residuals of the DL07 best fits plotted against the $500 \mu \mathrm{m}$ surface brightness $\Sigma_{500}$. As explained in the text, fractional residuals are defined as $\left(F_{v}-F_{\mathrm{DL} 07}\right) / F_{v} ; 250 \mu \mathrm{m}$ residuals are shown in the left panel, $350 \mu \mathrm{m}$ in the middle, and $500 \mu \mathrm{m}$ in the right. As in previous figures, data points are distinguished by $\mathrm{O} / \mathrm{H}$ with (red) circles showing $12+\log (\mathrm{O} / \mathrm{H})>8.4$, (green) triangles $8.0<12+\log (\mathrm{O} / \mathrm{H}) \leq 8.4$, and (blue) squares $12+\log (\mathrm{O} / \mathrm{H}) \leq 8.1$. Filled symbols correspond to positions with normalized (to optical radius $R_{\text {opt }}$ ) radii within $R / R_{\text {opt }} \leq 0.8$, and open symbols to larger radii. Unlike previous figures, here we show all data with $S / N \geq 3$ independently of their $\chi_{v}^{2}$; the DL07 fits with $\chi_{v}^{2} \leq 2$ are indicated by + . The (yellow) dashed lines give the linear regressions described in the text.

Ciesla et al. 2014). This is illustrated in Fig. 17 where we show separately the SEDs of the DL07 grain populations (silicates and carbonaceous grains). The dust in Fig. 17 is heated by a single radiation field, $U_{\min }$, with increasing $U_{\min }$ intensities associated with increasingly luminous SEDs (from $U_{\min }=0.1$ to 1 to 10 ). Lower $U_{\min }$ results in longer peak wavelengths. Thus, in addition to adding more cool dust to the SED, low $U_{\min }$ also implies that our $\lambda \leq 500 \mu \mathrm{m}$ data are not sampling well the Rayleigh-Jeans portion of the spectrum where the slope of the SED converges to the limiting value of $\beta+2$. This results in slopes that are apparently flatter with smaller values of $\beta$. Adding a more intense ISRF to $U_{\min }$, such as with a distribution of $U$ (e.g., Eq. (5)), would broaden the SED even more and move the peak to even shorter wavelengths. Second, the different chemical compositions of the grains themselves react differently to different $U_{\min }$, resulting in broader SEDs. As shown in Fig. 17, the peak wavelength changes with grain type, with silicate grains peaking toward longer wavelengths. The spread between the two peaks is larger for low $U_{\min }$ (lowest curve). Both the ISRF distribution and the different grain properties contribute to the shape of the dust SED, and conspire to cause the apparently flatter slopes $\beta$ associated with lower $U_{\min }$.

As a final check, because of previous suggestions that flatter $\beta$ may be associated with low metallicity (e.g., Galliano et al. 2011; Tabatabaei et al. 2014), we have looked for correlations of $\beta$ with metallicity in the KINGFISH sample. There is little evidence of such a correlation; $\beta$ at $12+\log (\mathrm{O} / \mathrm{H}) \lessgtr 8$ ranges from 0.5 to 3.0 , the same as its range at $12+\log (\mathrm{O} / \mathrm{H}) \gtrsim 8.5$.

Although small values of $\beta$ can be attributed to low $U_{\min }$ and temperature mixing in the form of more cool dust at low $T_{\text {dust }}$, large values of $\beta \gtrsim 2$ cannot be easily explained by such a phenomenon. Such high apparent values of $\beta$ may be due to real steepening of the dust SED in the FIR relative to the submm, with true $\beta \sim 2.5$, but flattened by temperature mixing along the LOS to slightly smaller $\beta$ values in the luminosity-weighted fits. If the emitting dust is indeed characterized by $\beta>2.5$ in the $100-500 \mu \mathrm{m}$ range, the interpretation is not clear. Laboratory studies of carbonaceous materials (Mennella et al. 1995, 1998) and various amorphous silicates (Agladze et al. 1996; Mennella et al. 1998; Coupeaud et al. 2011) generally find $\beta<2.2$ at
$T_{\text {dust }} \lesssim 30 \mathrm{~K}$ (except for sample E of Coupeaud et al. 2011, which had $\beta=2.5$ at $\left.T_{\text {dust }}=10 \mathrm{~K}\right)$. More work on dust emission with submm $(\lambda \gtrsim 800 \mu \mathrm{m})$ constraints is needed to better explore apparently high $\beta>2$ in nearby galaxies (e.g., Galametz et al. 2014).

\subsection{Model assessment and far-infrared deviations}

As briefly discussed in Sect. 4, the DL07 models roughly reproduce the SPIRE colors to within the uncertainties, but there may be systematic variations. Because of the importance of SPIRE wavelengths for understanding cold dust emission, and possibly constraining physical properties, in this section we explore the degree to which the SPIRE fluxes are well fit by the DL07 models. For each radial point, we have defined the SPIRE residual as $\left(F_{v}-F_{\mathrm{DL} 07}\right) / F_{v}$. Figure 18 shows these residuals at $250 \mu \mathrm{m}$, $350 \mu \mathrm{m}$, and $500 \mu \mathrm{m}$ plotted vs. $500 \mu \mathrm{m}$ surface brightness $\Sigma_{500}$. As in previous figures, the points are coded by $\mathrm{O} / \mathrm{H}$ with filled symbols corresponding to locations with $R / R_{\text {opt }} \leq 0.8$ and open ones to larger radii; moreover, only points with $S / N \geq 3$ are plotted. Unlike previous figures, in Fig. 18 (and Fig. 20) we have shown all the data with $S / N \geq 3$; DL07 fits with $\chi_{v}^{2} \leq 2$ are highlighted with + signs.

Even for the DL07 fits with $\chi_{v}^{2} \leq 2$, there are systematic variations of the residuals with $\Sigma_{500}$ as shown in Fig. 18. Over the range in $\Sigma_{500}$ shown in Fig. $18\left(0.1 \leq \Sigma_{500} \leq 10 \mathrm{MJy} \mathrm{sr}^{-1}\right)$, the SPIRE residuals are well correlated with $\Sigma_{500}$ at $350 \mu \mathrm{m}$ and $500 \mu \mathrm{m}$. However, at $250 \mu \mathrm{m}$, except for very low $\Sigma_{500}$ $\left(\lesssim 0.1 \mathrm{MJy} \mathrm{sr}^{-1}\right)$, the DL07 models well approximate the data; the best-fit slope for the residuals is 0.0 with an intercept of -0.014 . At $350 \mu \mathrm{m}$ and $500 \mu \mathrm{m}$, where the slopes are non-zero, the significance of the trend with $\Sigma_{500}$ is $>99.999 \%$ for DL07 fits with $\chi_{v}^{2} \leq 2$. However, the excursions are minor: at $350 \mu \mathrm{m}$, the mean residual is $\sim 3 \%$ positive at $\Sigma_{500}=0.1 \mathrm{MJy} \mathrm{sr}^{-1}$; at $500 \mu \mathrm{m}$, the mean residual is $\sim 9 \%$ at $\Sigma_{500}=0.1 \mathrm{MJy} \mathrm{sr}^{-1}$ and $\sim 6 \%$ at $\Sigma_{500}=0.3 \mathrm{MJy} \mathrm{sr}^{-1}$. Nevertheless, Fig. 18 shows that at low $500 \mu \mathrm{m}$ surface brightness, the DL07 fits tend to have $\chi_{v}^{2}>2$ at low $\Sigma_{500}$; there is increased scatter which may be related to metallicity (see below). 


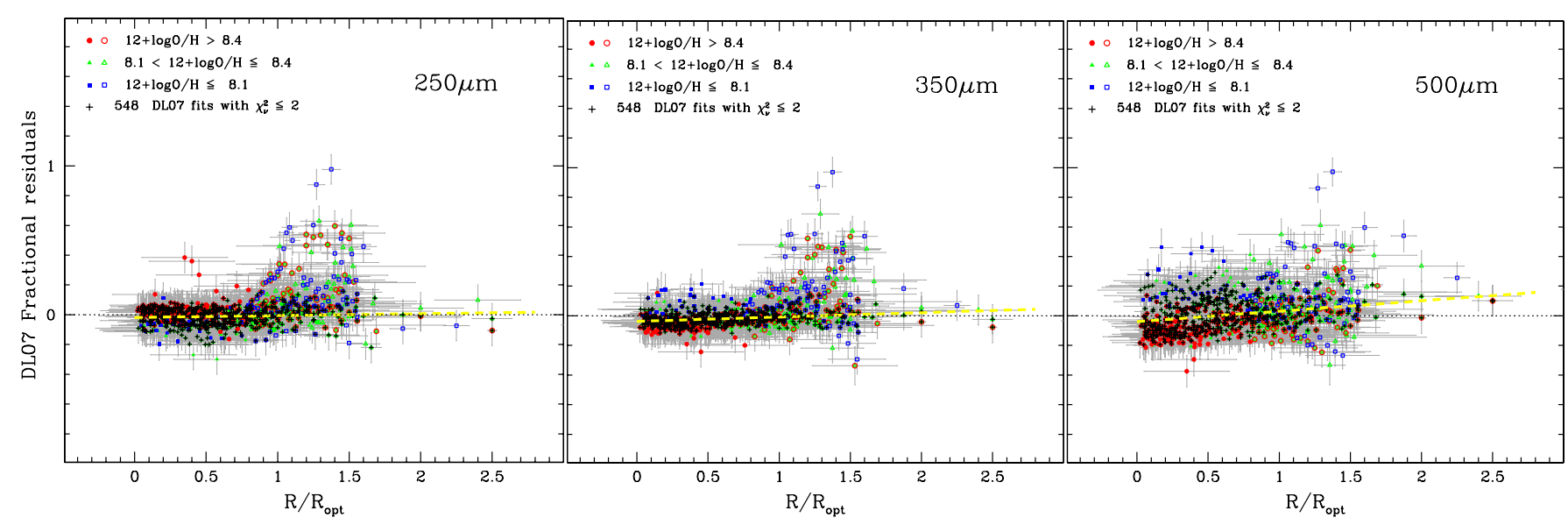

Fig. 19. SPIRE fractional residuals of the DL07 best fits plotted against the normalized radius, $R / R_{\text {opt }}$. As explained in the text, fractional residuals are defined as $\left(F_{v}-F_{\mathrm{DL} 07}\right) / F_{v} ; 250 \mu \mathrm{m}$ residuals are shown in the left panel, $350 \mu \mathrm{m}$ in the middle, and $500 \mu \mathrm{m}$ in the right. As in Fig. 18, data points are coded by $\mathrm{O} / \mathrm{H}$ with (red) circles showing $12+\log (\mathrm{O} / \mathrm{H})>8.4$, (green) triangles $8.0<12+\log (\mathrm{O} / \mathrm{H}) \leq 8.4$, and (blue) squares $12+\log (\mathrm{O} / \mathrm{H}) \leq 8.1$. Filled symbols correspond to positions with normalized (to optical radius $R_{\mathrm{opt}}$ ) radii within $R / R_{\mathrm{opt}} \leq 0.8$, and open symbols to larger radii. As in Fig. 18, we show all data with $S / N \geq 3$ independently of their $\chi_{v}^{2}$; the DL07 fits with $\chi_{v}^{2} \leq 2$ are indicated by + . The (yellow) dashed lines give the linear regressions described in the text.

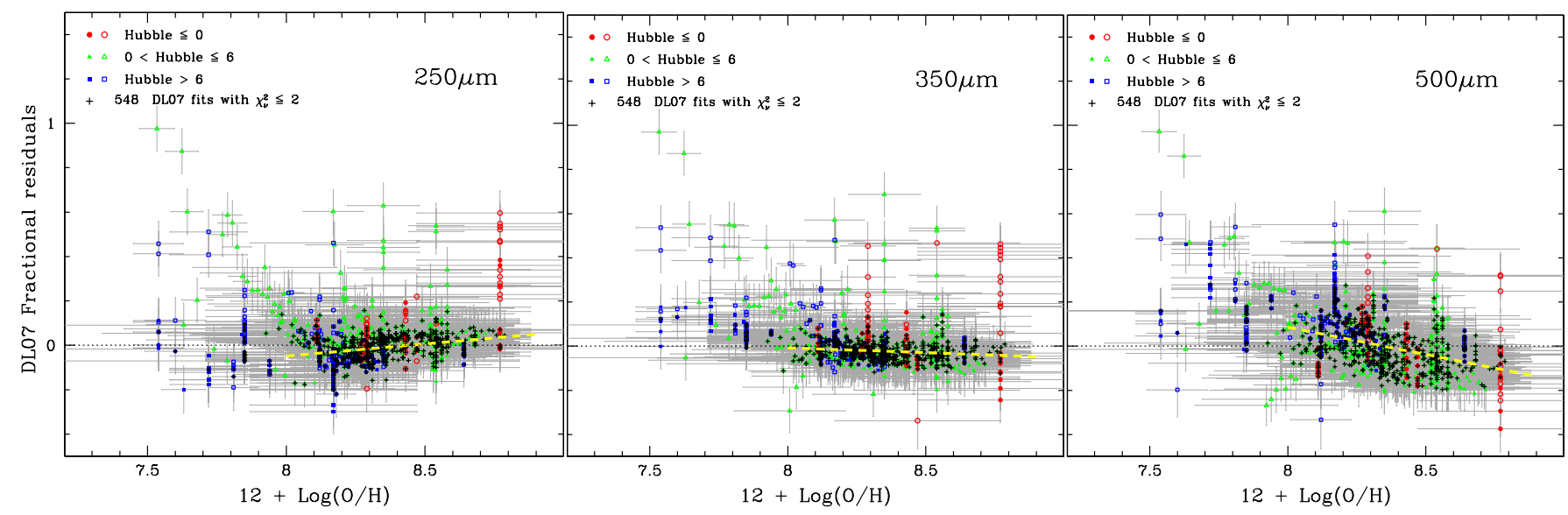

Fig. 20. SPIRE fractional residuals of the DL07 best fits plotted against the oxygen abundance, $12+\log (\mathrm{O} / \mathrm{H})$. As explained in the text, fractional residuals are defined as $\left(F_{v}-F_{\mathrm{DL} 07}\right) / F_{v} ; 250 \mu \mathrm{m}$ residuals are shown in the left panel, $350 \mu \mathrm{m}$ in the middle, and $500 \mu \mathrm{m}$ in the right. Data points are coded by Hubble type with (red) circles corresponding to early types $(T \leq 0)$, (green) triangles to spirals $(0<T \leq 0)$, and (blue) squares to late types $\left(T>6\right.$ ). Filled symbols correspond to positions with normalized (to optical radius $R_{\mathrm{opt}}$ ) radii within $R / R_{\mathrm{opt}} \leq 0.8$, and open symbols to larger radii. As in Fig. 18 all points with $S / N \geq 3$ are plotted, with the DL07 fits with $\chi_{v}^{2} \leq 2$ shown by + . The (yellow) dashed lines give the linear regressions described in the text.

Similar trends are seen in Fig. 19 where we have plotted residuals against normalized optical radius, $R / R_{\mathrm{opt}}$. The DL07 models follow the data well at $250 \mu \mathrm{m}$ with a regression slope of 0.0 . However, as before, at $350 \mu \mathrm{m}$ and $500 \mu \mathrm{m}$, the trends are highly significant $(>99.999 \%$ for DL07 fits with $\chi_{v}^{2} \leq 2$ ) but with minimal excursions: at $R_{\mathrm{opt}}=1.5, \lessgtr 1 \%$ and $\sim 7 \%$ at $350 \mu \mathrm{m}$ and $500 \mu \mathrm{m}$, respectively. The trends with $R / R_{\text {opt }}$ are probably reflecting those with surface brightness $\Sigma_{500}$ because of the tendency for low surface brightnesses to occur in the outer radii of galaxies.

Figure 20 gives the same DL07 residuals as in Figs. 18 and 19 but plotted against oxygen abundance, $12+\log (\mathrm{O} / \mathrm{H})$. As before, there are systematic trends revealed by highly significant $(>99.999 \%)$ correlations between SPIRE residuals and O/H. For $12+\log (\mathrm{O} / \mathrm{H})=8$, the mean residual is $\sim 5 \%$ negative at $250 \mu \mathrm{m}$, and $\sim 9 \%$ positive at $500 \mu \mathrm{m}$. Interestingly, large $350 \mu \mathrm{m}$ residuals are spread over a large range in $\mathrm{O} / \mathrm{H}$, unlike those with respect to $\Sigma_{500}$ which are large only for $\Sigma_{500} \lesssim 0.1 \mathrm{MJy} \mathrm{sr}^{-1}$. Moreover, there is no linear trend of the $350 \mu$ m residuals relative to $\mathrm{O} / \mathrm{H}$, unlike for those with respect to $\Sigma_{500}$. This could be an indication that the DL07 best fits are trying to split the differences for $\mathrm{O} / \mathrm{H}$ among the SPIRE bands; thus there naturally would be a deficit at $250 \mu \mathrm{m}$, neutrality at $350 \mu \mathrm{m}$, and a positive excess at $500 \mu \mathrm{m}$.

If the DL07 dust models were adjusted by these minute amounts at $12+\log (\mathrm{O} / \mathrm{H})=8(5 \%$ smaller at $250 \mu \mathrm{m}, 1 \%$ smaller at $350 \mu \mathrm{m}, \sim 9 \%$ larger at $500 \mu \mathrm{m})$, the SPIRE colors shown in Fig. 4 would be shifted $\sim 4 \%$ down and $\sim 10 \%$ to the left, toward the apparent $\beta \sim 1$ curve, which would be roughly appropriate for low metallicity. Correcting for $\Sigma_{500}$, the shifts would be of similar amplitude, again consistent with $\beta \sim 1$. If instead, we consider the metal-rich and high $\Sigma_{500}$ adjustments, the trends are of similar amplitude but opposite sign, thus moving the colors $\sim 5 \%$ up and $\sim 3 \%$ to the right, toward the apparent $\beta \sim 2$ curve. Adjusting the DL07 models by the small corrections suggested by the mean DL07 SPIRE residuals would bring the models to better agreement with the data. 


\section{6. $500 \mu \mathrm{m}$ excess}

Because the DL07 models are constrained at SPIRE wavelengths only indirectly through grain properties, the agreement between the models and the data is quite good. Nevertheless, at low metallicities and low surface brightnesses, the residuals at $500 \mu \mathrm{m}$ are slightly positive $(\$ 9 \%)$ at a high significance level. If we consider the DL07 fits with $\chi_{v}^{2}>2$, the excesses at all SPIRE wavelengths are even larger, although it is difficult to define systematic trends. Other work has also found evidence of a $500 \mu \mathrm{m}$ excess in low-metallicity systems such as the LMC and dwarf galaxies (e.g., Gordon et al. 2010; Galliano et al. 2011; Galametz et al. 2011). Ciesla et al. (2014) find a similar trend in the Herschel Reference Survey with the DL07 models underestimating the $500 \mu \mathrm{m}$ flux for low-mass systems (which would be related to low metallicity). We conclude that in the KINGFISH profiles there is evidence of a very weak submm excess, $\lesssim 10 \%$, at low surface brightnesses and at low metallicity (c.f., Kirkpatrick et al. 2013). Because of metallicity gradients, and the resulting interdependence of metallicity and surface brightness in spiral disks, a partial correlation analysis would be necessary to establish whether the excess results primarily from low metallicity or from low surface brightness. Longer wavelength data are needed (e.g., Galametz et al. 2014) to establish the existence of a systematic submm excess and the degree to which it depends on the grain properties of the models.

\section{Summary and conclusions}

We have analyzed the entire collection of radial surface brightness profiles for $61 \mathrm{KINGFISH}$ galaxies both in terms of radial trends and SED properties. By fitting the radial profiles with exponentials, we find that the $250 \mu \mathrm{m}$ scalelength is on average comparable to that of the stars, as measured by the $3.6 \mu \mathrm{m}$ scalelength. In the KINGFISH galaxies, except for isolated cases, the dust tends to be distributed in the same way as the stars.

We have also fitted the SEDs of each annular region with single-temperature MBB and DL07 models. To better understand the relation between physical parameters of dust emission and the apparent $T_{\text {dust }}$ and emissivity index $\beta$, the best-fit DL07 models themselves have also been fit with singletemperature MBB models. The analysis of the radial trends of these parameters shows that dust temperature $T_{\text {dust }}$, dust optical depth $\tau_{\text {dust }}$, and $U_{\min }$ all tend to decrease with radius. The PDR fraction, $f_{\mathrm{PDR}}$, shows a slight increase at large radii, perhaps indicating the presence of extended UV disks in some galaxies that could be responsible for PDR-like emission.

The analysis of the MBBV fits to the DL07 models shows that the models are well able to reproduce flat spectral slopes with $\beta \lesssim 1$. Our methodology for the MBB fitting to some extent mitigates the usual correlation or degeneracy between $T_{\text {dust }}$ and $\beta$, and through an analysis of $\tau_{\text {dust }}$ and temperature binning, we find that shallow slopes $(\beta \lesssim 1)$ in the data are associated with cool $T_{\text {dust }}$ in a similar way as the DL07 models. Our results also show that the minimum ISRF intensity, $U_{\min }$, responsible for heating the bulk of the dust in most galaxies is closely related to the apparent emissivity index $\beta$, with lower $U_{\min }$ associated with flatter $\beta$. Hence, we conclude that temperature mixing is a major cause of trends of $\beta$ commonly seen in MBB fitting of IR SEDs of galaxies. Temperature mixing may arise from the distribution of ISRF intensities responsible for heating the dust, or from the different properties of the grain populations, or both. It is therefore difficult to ascribe variations in $\beta$ to real physical properties of dust grains.
Finally, we assess the ability of the DL07 models to fit the observed SPIRE fluxes, and find generally very good agreement. However, there is some evidence of a small $500 \mu \mathrm{m}$ excess, $\sim 10 \%$, for regions of low dust surface brightness and low metallicity.

The detailed study of dust emission and grain properties in galaxies is still in its infancy. More work is needed at high spatial resolution and long wavelengths to establish whether or not current dust models are able to accommodate the observations in physical regimes that are more extreme than those usually encountered in the disks of spiral galaxies.

Acknowledgements. We dedicate this paper to the memory of Charles W. Engelbracht, whose excellence and commitment as a scientist were fundamental for this work, and without whom the SPIRE images analyzed here would not have existed. We thank the anonymous referee for concise comments which improved the clarity of the paper. S.B. and L.K.H. acknowledge support from PRIN-INAF 2012/13. B.T.D. was supported in part by NSF grant AST-1408723. Use was made of the NASA/IPAC Extragalactic Database (NED).

\section{References}

Agladze, N. I., Sievers, A. J., Jones, S. A., Burlitch, J. M., \& Beckwith, S. V. W. 1996, ApJ, 462, 1026

Alatalo, K., Blitz, L., Young, L. M., et al. 2011, ApJ, 735, 88

Alton, P. B., Trewhella, M., Davies, J. I., et al. 1998, A\&A, 335, 807

Amblard, A., Riguccini, L., Temi, P., et al. 2014, ApJ, 783, 135

Aniano, G., Draine, B. T., Calzetti, D., et al. 2012, ApJ, 756, 138

Auld, R., Bianchi, S., Smith, M. W. L., et al. 2013, MNRAS, 428, 1880

Begum, A., Chengalur, J. N., Karachentsev, I. D., Kaisin, S. S., \& Sharina, M. E. 2006, MNRAS, 365, 1220

Bendo, G. J., Wilson, C. D., Pohlen, M., et al. 2010, A\&A, 518, L65

Bendo, G. J., Boselli, A., Dariush, A., et al. 2012, MNRAS, 419, 1833

Bernard, J. P., Abergel, A., Ristorcelli, I., et al. 1999, A\&A, 347, 640

Bianchi, S. 2007, A\&A, 471, 765

Bianchi, S. 2013, A\&A, 552, A89

Bianchi, S., \& Xilouris, E. M. 2011, A\&A, 531, L11

Bigiel, F., Leroy, A., Walter, F., et al. 2008, AJ, 136, 2846

Bigiel, F., Leroy, A., Seibert, M., et al. 2010, ApJ, 720, L31

Boquien, M., Calzetti, D., Combes, F., et al. 2011, AJ, 142, 111

Boselli, A., Eales, S., Cortese, L., et al. 2010, PASP, 122, 261

Boselli, A., Ciesla, L., Cortese, L., et al. 2012, A\&A, 540, A54

Bosma, A. 1981, AJ, 86, 1791

Ciesla, L., Boquien, M., Boselli, A., et al. 2014, A\&A, 565, A128

Compiègne, M., Verstraete, L., Jones, A., et al. 2011, A\&A, 525, A103

Cortese, L., Boissier, S., Boselli, A., et al. 2012, A\&A, 544, A101

Cortese, L., Fritz, J., Bianchi, S., et al. 2014, MNRAS, 440, 942

Coupeaud, A., Demyk, K., Meny, C., et al. 2011, A\&A, 535, A124

Crosthwaite, L. P., Turner, J. L., \& Ho, P. T. P. 2000, AJ, 119, 1720

Croxall, K. V., van Zee, L., Lee, H., et al. 2009, ApJ, 705, 723

Daigle, O., Carignan, C., Amram, P., et al. 2006, MNRAS, 367, 469

Dale, D. A., \& Helou, G. 2002, ApJ, 576, 159

Dale, D. A., Giovanelli, R., Haynes, M. P., et al. 1997, AJ, 114, 455

Dale, D. A., Helou, G., Contursi, A., Silbermann, N. A., \& Kolhatkar, S. 2001, ApJ, 549, 215

Dale, D. A., Cohen, S. A., Johnson, L. C., et al. 2009, ApJ, 703, 517

Dale, D. A., Aniano, G., Engelbracht, C. W., et al. 2012, ApJ, 745, 95

Davies, R. I., Maciejewski, W., Hicks, E. K. S., et al. 2009, ApJ, 702, 114

de Blok, W. J. G., Walter, F., Brinks, E., et al. 2008, AJ, 136, 2648

De Geyter, G., Baes, M., Fritz, J., \& Camps, P. 2013, A\&A, 550, A74

Draine, B. T., \& Li, A. 2001, ApJ, 551, 807

Draine, B. T., \& Li, A. 2007, ApJ, 657, 810

Draine, B. T., Aniano, G., Krause, O., et al. 2014, ApJ, 780, 172

Engelbracht, C. W., Hunt, L. K., Skibba, R. A., et al. 2010, A\&A, 518, L56

Fazio, G. G., Hora, J. L., Allen, L. E., et al. 2004, ApJS, 154, 10

Finkbeiner, D. P., Davis, M., \& Schlegel, D. J. 1999, ApJ, 524, 867

Freeman, K. C. 1970, ApJ, 160, 811

Galametz, M., Madden, S. C., Galliano, F., et al. 2011, A\&A, 532, A56

Galametz, M., Kennicutt, R. C., Albrecht, M., et al. 2012, MNRAS, 425, 763

Galametz, M., Albrecht, M., Kennicutt, R., et al. 2014, MNRAS, 439, 2542

Galliano, F., Hony, S., Bernard, J.-P., et al. 2011, A\&A, 536, A88

Gil de Paz, A., Madore, B. F., Boissier, S., et al. 2005, ApJ, 627, L29

Giovanelli, R., Haynes, M. P., Salzer, J. J., et al. 1994, AJ, 107, 2036

Gordon, K. D., Galliano, F., Hony, S., et al. 2010, A\&A, 518, L89

Gordon, K. D., Meixner, M., Meade, M. R., et al. 2011, AJ, 142, 102 
Gordon, K. D., Roman-Duval, J., Bot, C., et al. 2014, ApJ, 797, 85 Griffin, M. J., Abergel, A., Abreu, A., et al. 2010, A\&A, 518, L3 Griffin, M. J., North, C. E., Schulz, B., et al. 2013, MNRAS, 434, 992 Groves, B., Krause, O., Sandstrom, K., et al. 2012, MNRAS, 426, 892 Haas, M., Lemke, D., Stickel, M., et al. 1998, A\&A, 338, L33

Heckman, T. M., Lehnert, M. D., Strickland, D. K., \& Armus, L. 2000, ApJS, 129,493

Hildebrand, R. H. 1983, QJRAS, 24, 267

Holmberg, E. 1958, Meddelanden fran Lunds Astronomiska Observatorium Serie II, 136, 1

Holwerda, B. W., González, R. A., van der Kruit, P. C., \& Allen, R. J. 2005, A\&A, 444, 109

Holwerda, B. W., Bianchi, S., Böker, T., et al. 2012a, A\&A, 541, L5

Holwerda, B. W., Pirzkal, N., \& Heiner, J. S. 2012b, MNRAS, 427, 3159

Hubble, E. P. 1926, ApJ, 64, 321

Hunt, L. K., Pierini, D., \& Giovanardi, C. 2004, A\&A, 414, 905

Juvela, M., \& Ysard, N. 2012, A\&A, 541, A33

Juvela, M., Montillaud, J., Ysard, N., \& Lunttila, T. 2013, A\&A, 556, A63

Karim, A., Schinnerer, E., Martínez-Sansigre, A., et al. 2011, ApJ, 730, 61

Kelly, B. C., Shetty, R., Stutz, A. M., et al. 2012, ApJ, 752, 55

Kennicutt, R. C., Jr., Armus, L., Bendo, G., et al. 2003, PASP, 115, 928

Kennicutt, R. C., Calzetti, D., Aniano, G., et al. 2011, PASP, 123, 1347

Kirkpatrick, A., Calzetti, D., Galametz, M., et al. 2013, ApJ, 778, 51

Kirkpatrick, A., Calzetti, D., Galametz, M., et al. 2014, ApJ, submitted

Kreckel, K., Armus, L., Groves, B., et al. 2014, ApJ, 790, 26

Laureijs, R. J., Watson, D., Metcalfe, L., et al. 2000, A\&A, 359, 900

Leroy, A. K., Walter, F., Brinks, E., et al. 2008, AJ, 136, 2782

Li, A, \& Draine, B. T. 2001, ApJ, 554, 778

Li, Y., Bresolin, F., \& Kennicutt, R. C., Jr. 2013, ApJ, 766, 17

Mathis, J. S., Mezger, P. G., \& Panagia, N. 1983, A\&A, 128, 212

Mennella, V., Colangeli, L., \& Bussoletti, E. 1995, A\&A, 295, 165

Mennella, V., Brucato, J. R., Colangeli, L., et al. 1998, ApJ, 496, 1058

Meny, C., Gromov, V., Boudet, N., et al. 2007, A\&A, 468, 171

Moriondo, G., Giovanardi, C., \& Hunt, L. K. 1998, A\&AS, 130, 81

Moustakas, J., \& Kennicutt, R. C., Jr. 2006, ApJ, 651, 155

Moustakas, J., Kennicutt, R. C., Jr., Tremonti, C. A., et al. 2010, ApJS, 190, 233

Muñoz-Mateos, J. C., Gil de Paz, A., Boissier, S., et al. 2007, ApJ, 658, 1006

Muñoz-Mateos, J. C., Gil de Paz, A., Boissier, S., et al. 2009a, ApJ, 701, 1965

Muñoz-Mateos, J. C., Gil de Paz, A., Zamorano, J., et al. 2009b, ApJ, 703, 1569

Muñoz-Mateos, J. C., Sheth, K., Gil de Paz, A., et al. 2013, ApJ, 771, 59

Murphy, E. J., Helou, G., Kenney, J. D. P., Armus, L., \& Braun, R. 2008, ApJ, 678,828

Nguyen, H. T., Schulz, B., Levenson, L., et al. 2010, A\&A, 518, L5

Noeske, K. G., Weiner, B. J., Faber, S. M., et al. 2007, ApJ, 660, L43

Oh, S.-H., de Blok, W. J. G., Walter, F., Brinks, E., \& Kennicutt, R. C., Jr. 2008, AJ, 136, 2761

Ott, S. 2010, ASP Conf. Ser., 434, 139

Paradis, D., Bernard, J.-P., \& Mény, C. 2009, A\&A, 506, 745

Paradis, D., Paladini, R., Noriega-Crespo, A., et al. 2012, A\&A, 537, A113

Pellegrini, E. W., Smith, J. D., Wolfire, M. G., et al. 2013, ApJ, 779, L19

Pilbratt, G. L., Riedinger, J. R., Passvogel, T., et al. 2010, A\&A, 518, L1

Pilyugin, L. S., \& Thuan, T. X. 2005, ApJ, 631, 231

Pilyugin, L. S., Thuan, T. X., \& Vílchez, J. M. 2007, MNRAS, 376, 353

Planck Collaboration XVII. 2011, A\&A, 536, A17

Planck Collaboration XXII. 2011, A\&A, 536, A22

Planck Collaboration XXIII. 2011, A\&A, 536, A23

Planck Collaboration XXIV. 2011, A\&A, 536, A24

Planck Collaboration Int. XIV. 2014, A\&A, 564, A45

Poglitsch, A., Waelkens, C., Geis, N., et al. 2010, A\&A, 518, L2

Pollack, J. B., Hollenbach, D., Beckwith, S., et al. 1994, ApJ, 421, 615

Rieke, G. H., Young, E. T., Engelbracht, C. W., et al. 2004, ApJS, 154, 25

Roussel, H. 2013, PASP, 125, 1126

Roussel, H., Helou, G., Smith, J. D., et al. 2006, ApJ, 646, 841

Salim, S., Rich, R. M., Charlot, S., et al. 2007, ApJS, 173, 267

Sauvage, M., \& Thuan, T. X. 1992, ApJ, 396, L69

Shetty, R., Kauffmann, J., Schnee, S., \& Goodman, A. A. 2009a, ApJ, 696, 676

Shetty, R., Kauffmann, J., Schnee, S., Goodman, A. A., \& Ercolano, B. 2009b, ApJ, 696, 2234
Skibba, R. A., Engelbracht, C. W., Dale, D., et al. 2011, ApJ, 738, 89 Smith, M. W. L., Eales, S. A., Gomez, H. L., et al. 2012, ApJ, 756, 40 Stepnik, B., Abergel, A., Bernard, J.-P., et al. 2003, A\&A, 398, 551

Storchi-Bergmann, T., Calzetti, D., \& Kinney, A. L. 1994, ApJ, 429, 572

Tabatabaei, F. S., Braine, J., Xilouris, E. M., et al. 2014, A\&A, 561, A95

Tamburro, D., Rix, H.-W., Walter, F., et al. 2008, AJ, 136, 2872

Vader, J. P., Frogel, J. A., Terndrup, D. M., \& Heisler, C. A. 1993, AJ, 106, 1743

Verstappen, J., Fritz, J., Baes, M., et al. 2013, A\&A, 556, A54

Walter, F., Brinks, E., de Blok, W. J. G., et al. 2008, AJ, 136, 2563

Weingartner, J. C., \& Draine, B. T. 2001, ApJ, 548, 296

Williams, B. F., Dalcanton, J. J., Dolphin, A. E., Holtzman, J., \& Sarajedini, A. 2009, ApJ, 695, L15

Xilouris, E. M., Byun, Y. I., Kylafis, N. D., Paleologou, E. V., \& Papamastorakis, J. 1999, A\&A, 344, 868

Ysard, N., Juvela, M., Demyk, K., et al. 2012, A\&A, 542, A21

Zibetti, S., \& Groves, B. 2011, MNRAS, 417, 812

1 INAF-Osservatorio Astrofisico di Arcetri, Largo E. Fermi 5, 50125 Firenze, Italy e-mail: hunt@arcetri.astro.it

2 Department of Astrophysical Sciences, Princeton University, Princeton, NJ 08544, USA

3 Space Telescope Science Institute, 3700 San Martin Drive, Baltimore, MD 21218, USA

4 Sterrenkundig Observatorium, Universiteit Gent, Gent, Belgium

5 Institut d'Astrophysique Spatiale, bâtiment 121, Université Paris-Sud 11, CNRS UMR 8617, 91405 Orsay, France

6 Department of Astronomy, University of Massachusetts, Amherst, MA 01003, USA

7 Department of Physics and Astronomy, University of Wyoming, Laramie, WY 82071, USA

8 NASA Herschel Science Center, IPAC, California Institute of Technology, Pasadena, CA 91125, USA

9 Steward Observatory, University of Arizona, 933 North Cherry Avenue, Tucson, AZ 85721, USA

10 Institute of Astronomy, University of Cambridge, Madingley Road, Cambridge CB3 OHA, UK

11 Institut d'Astrophysique de Paris, Sorbonne Universités, UPMC Univ. Paris 06, CNRS UMR 7095, 75014 Paris, France

12 Department of Physics and Astronomy, McMaster University, Hamilton, ON L8S 4M1, Canada

13 Department of Astronomy and Laboratory for Millimeter-wave Astronomy, University of Maryland, College Park, MD 20742, USA

14 Institute of Astronomy, University of Cambridge, Madingley Road, Cambridge CB3 0HA, UK

15 Department of Astronomy, The Ohio State University, 140 West 18th Avenue, Columbus OH 43210, USA

16 European Southern Observatory, Karl-Schwarzchild-Str. 2, 85748 Garching-bei-Mnchen, Germany

17 Departamento de Astrofísica, Universidad Complutense de Madrid, Avda. de la Complutense s/n, 28040 Madrid, Spain

18 Department of Physics and Astronomy, SUNY Stony Brook, Stony Brook NY 11794-3800, USA

19 European Southern Observatory, Casilla 19001, Santiago 19, Chile

20 Max-Planck-Institut fur Astronomie, Konigstuhl 17, 69117 Heidelberg, Germany

21 Steward Observatory, University of Arizona, 933 N. Cherry Ave, Tucson AZ 85721, USA

22 CEA, Laboratoire AIM, Irfu/SAp, Orme des Merisiers, 91191 Gif-sur-Yvette, France

23 Institut d'Astrophysique de Paris, UMR 7095 CNRS, Université Pierre et Marie Curie, 75014 Paris, France 